\title{
Adiabatic continuity and confinement in supersymmetric Yang-Mills theory on the lattice
}

\author{
Georg Bergner, ${ }^{a}$ Stefano Piemonte ${ }^{b}$ and Mithat Ünsal ${ }^{c}$ \\ ${ }^{a}$ Friedrich-Schiller-University Jena, Institute of Theoretical Physics, \\ Max-Wien-Platz 1, D-07743 Jena, Germany \\ ${ }^{b}$ University of Regensburg, Institute for Theoretical Physics, \\ Universitätsstr. 31, D-93040 Regensburg, Germany \\ ${ }^{c}$ Department of Physics, North Carolina State University, \\ Raleigh, NC 27695, U.S.A. \\ E-mail: georg.bergner@uni-jena.de, stefano.piemonte@ur.de, \\ unsal.mithat@gmail.com
}

ABSTRACT: This work is a step towards merging the ideas that arise from semi-classical methods in continuum QFT with analytic/numerical lattice field theory. In this context, we consider Yang-Mills theories coupled to fermions transforming in the adjoint representation of the gauge group. These theories have the remarkable property that confinement and discrete chiral symmetry breaking can persist at weak coupling on $\mathbb{R}^{3} \times S^{1}$ up to small (nonthermal) compactification radii. This work presents a lattice investigation of a gauge theory coupled to a single adjoint Majorana fermion, the $\mathcal{N}=1$ Supersymmetric Yang-Mills theory (SYM), and opens the prospect to understand analytically a number of non-perturbative phenomena, such as confinement, mass gap, chiral and center symmetry realizations, both on the lattice and in the continuum. We study the compactification of $\mathcal{N}=1 \mathrm{SYM}$ on the lattice with periodic and thermal boundary conditions applied to the fermion field. We provide numerical evidences for the conjectured absence of phase transitions with periodic boundary conditions for sufficiently light lattice fermions (stability of center-symmetry), for the suppression of the chiral transition, and we provide also a diagnostic for Abelian vs. non-Abelian confinement, based on per-site Polyakov loop eigenvalue distribution functions. We identify the role of the lattice artefacts that become relevant in the very small radius regime, and we resolve some puzzles in the naive comparison between continuum and lattice.

Keywords: Confinement, Lattice Quantum Field Theory, Supersymmetric Gauge Theory, Wilson, 't Hooft and Polyakov loops

ARXIV EPRINT: 1806.10894 


\section{Contents}

1 Introduction 1

2 Adjoint QCD on the lattice 4

3 Order parameters for the phase diagram of adjoint QCD 6

4 The perturbative effective potential for the Polyakov loop on the lattice 7

4.1 Abelian vs. non-Abelian confinement regimes: first pass 12

$\begin{array}{lll}4.2 & \text { Different Polyakov line effective actions } & 13\end{array}$

5 The phase diagram on the continuum and lattice $\quad 14$

$\begin{array}{ll}5.1 \text { Explanation of the discrepancy of lattice and continuum } & 18\end{array}$

6 Numerical results for compactified adjoint QCD 20

6.1 Confined and deconfined phases in SYM theory 21

$6.2 N_{f}=2$ QCD(adj) 23

6.3 Eigenvalue distributions and Abelian vs. non-abelian confinement 25

6.4 The Polyakov loop in the adjoint representation 28

$\begin{array}{ll}\text { 6.5 The chiral condensate } & 29\end{array}$

$\begin{array}{lll}7 & \text { Conclusions } & 30\end{array}$

\section{Introduction}

Confinement is a feature of strong interactions emerging in the long distance physics of certain non-Abelian gauge theories. The effective low energy degrees of freedom of these theories are colorless bound states which cannot be described as a simple combination of the elementary fields. The non-perturbative features of the strongly interacting regime, like confinement and the bound state spectrum, are consequently still beyond any analytical understanding. Perturbation theory can provide a reliable approximation of scattering processes only at very high energy, but it cannot explain the structure and the properties of low energy states. Despite the success of the numerical lattice simulations in reproducing the observed hadron masses, an understanding of the nature of confinement is still missing on $\mathbb{R}^{4}$.

An interesting alternative analytical approach is based on a controlled semiclassical analysis that tries to identify the most relevant field contributions as in the Polyakov model on $\mathbb{R}^{3}[1]$. However, despite the important successes of the Polyakov model, the idea remained dormant in QCD in four dimensions, mainly due to two reasons: one is that it was often believed that the Polyakov mechanism was a manifestly three-dimensional 
mechanism, and the other is that in theories with exactly massless fermions, the Polyakov mechanism would not work [2]. About ten years ago, new ideas and techniques in semiclassics started to emerge and the utility of (justified) semi-classics into non-perturbative problems in QCD and other vector-like and chiral gauge theories has been realized [3-6]. For related earlier work, see [7-10].

More recently theories with matter fields transforming in the adjoint representation of the gauge group and certain supersymmetric theories became a valuable subject of these investigations [11, 12] using tools of resurgence, and Picard-Lefschetz theory. The QFT application of resurgence may potentially provide a rigorous non-perturbative definition of path integral in continuum QFT. At least, in certain quantum mechanical models with instantons, it is proven that path integral can be decoded into a full semi-classical resurgent expansion [13], and in certain asymptotically free two-dimensional QFTs, such as twodimensional $\mathbb{C P}^{N-1}$ and principal chiral models, ideas related to resurgence have been fruitful to partially resolve the renormalon problem [14-16].

The semiclassical analysis corresponds to a sum of the expansions around the dominant field configurations in a regime where weak coupling methods are reliable. (See [14] for a recent review.) The inclusion of non-trivial configurations should overcome the limitation of standard perturbation theory by incorporating exponentially small non-perturbative effects of the form $e^{-A /\left(g^{2} N\right)}$ where $A$ is pure number and $\left(g^{2} N\right)$ is the 't Hooft coupling. ${ }^{1}$

If the time dimension is compactified and the length of the compact dimension $R$ is smaller than the strong scale $\Lambda^{-1}$, the theory becomes weakly coupled due to asymptotic freedom. In this regime, QCD and Yang-Mills theories are in the deconfined phase as can be shown by studying the Gross-Pisarski-Yaffe (GPY) potential [17]. Consequently this region is disconnected by a phase transition (or crossover) from the large $R$ low energy confined phase that one aims to understand. Furthermore, the dynamics of the theory at distances much larger than $R$ is governed by a $3 \mathrm{~d}$ pure Yang-Mills theory, which develops the strong (magnetic) scale on its own.

The situation is different in a gauge theory where the fermion fields are in the adjoint representation and fulfill periodic boundary conditions in the compact direction. Periodic boundary condition in path integral formalism map to

$$
\tilde{Z}(R, m)=\operatorname{tr}\left[e^{-R \hat{H}(m)}(-1)^{F}\right]
$$

in the operator formalism, where $\hat{H}(m)$ is the Hamiltonian operator and $m$ is the fermion mass. This trace is a graded state sum which assigns an over-all $(+1)$ sign to boson states and (-1) to fermion states. Note that (1.1) for $N_{f}=1$ and $m=0$ is the well-known Witten index [18]. For general $N_{f}$ or $m \neq 0$, the trace is a (non-thermal) twisted partition function which probes the phase structure of the theory as a function of $R[4,19]$.

If we consider the one-loop GPY potential with this partition function, the centerdestabilizing potential induced by gauge bosons is overwhelmed by the center stabilizing contributions from sufficiently light fermion fields [20,21]. In this case, the small $R$ regime

\footnotetext{
${ }^{1}$ Note that this type of non-perturbative effect is exponentially larger than the usual four-dimensional instanton effects in gauge theories, which are of the form $e^{-A / g^{2}}$.
} 
is in a center symmetric phase on $\mathbb{R}^{3} \times S^{1}$ which is non-perturbatively calculable by semiclassical methods. The theory exhibits the semiclassical magnetic bion mechanism of confinement, a non-perturbative mass gap for gauge fluctuations, and, if it exists in the theory, a discrete chiral symmetry breaking.

The small $R$ regime on $\mathbb{R}^{3} \times S^{1}$ provides a controlled semi-classical approximation in sharp distinction from the so-called dilute instanton gas picture on $\mathbb{R}^{4}$ which is an uncontrolled approximation, see section "the uses of instantons" in [22] and [23] for the limitations of the latter approach. The calculable semi-classical regime might in certain cases be smoothly connected to the confined strongly coupled regime at large $R$ [4-6, 24]. This is the notion of adiabatic continuity which posits that a semi-classical calculable regime, under appropriate conditions, may be smoothly connected to a strong coupling regime without any intermediate phase transitions.

An exact cancellation between fermion and boson perturbative contributions occurs in the particular case of the $\mathcal{N}=1$ Supersymmetric Yang-Mills theory (SYM) or $N_{f}=1$ QCD(adj) [10, 25]. Both center-stability as well as confinement of compactified SYM are solely due to non-trivial semiclassical contributions, namely neutral and magnetic bions. The phase structure of gauge theories with $N_{f}$ adjoint Majorana fermions, $N_{f}$-flavour $\mathrm{QCD}(\mathrm{adj})$, opens therefore a promising perspective for the understanding of the mechanisms of confinement $[4,11]$, see also [26-28].

A regularization providing a-priori non-perturbative numerical simulations is required to prove this continuous connection of the small $R$ regime to the strongly coupled large $R$ confined phase and to verify the absence of intermediate phase transitions. Monte-Carlo lattice simulations are an ideal first principles method from this point of view.

The first observation of the absence of deconfinement in compactified supersymmetric Yang-Mills theory has been presented in an earlier publication [29], we are investigating the phases of the theory more closely in this work. In order to understand the influence of the lattice discretization, we first compare the perturbative analysis in the continuum and on the lattice. It turns out that the discretization of the fermion action on the lattice is of particular importance in the small $R$ regime. Due to lattice artefacts, the exact cancellation between fermion and boson contributions can only be achieved in the continuum limit. Nevertheless we show that qualitative features of the semiclassical predictions are reproduced on the lattice. In contrast to our earlier investigations we concentrate here on a fixed scale approach which avoids additional complications introduced by a variation of the lattice spacing. Part of the work is done with a clover improved Wilson fermion action in order to reduce the discretization effects. We consider the order parameters for the chiral and the deconfinement transition. The primary focus is on supersymmetric Yang-Mills theory, but we have also investigated the $N_{f}$ dependence in a numerical study of $N_{f}=2 \mathrm{QCD}(\operatorname{adj})$.

We introduce the lattice formulation and the order parameters for the investigations of the phase transitions in the next two sections. In section 4 we provide a detailed discussion of the perturbative effective potential of the Polyakov loop on the lattice and in the continuum. Based on the analysis of the effective potential, we derive predictions of the phase diagram of $\mathrm{QCD}(\mathrm{adj})$ in the weak coupling regime and conjectures about the general phase diagram in section 5 . After these theoretical considerations, we present our 
numerical results in section 6 . The signals for deconfinement are investigated in $N_{f}=1$ and $N_{f}=2 \mathrm{QCD}$ (adj). In addition we present results for the per-site constraint effective potential of the Polyakov line phase, the adjoint Polyakov loop, and the chiral condensate in the $N_{f}=1$ case.

\section{Adjoint QCD on the lattice}

$\mathrm{QCD}(\operatorname{adj})$ consists of a non-Abelian gauge-field $A_{\mu}^{c}(x)$ minimally coupled to $N_{f}$ Majorana fermions $\lambda_{i}^{c}(x)$. The expression of the continuum action is similar to QCD

$$
S=\int d^{4} x\left\{-\frac{1}{4} F_{\mu \nu} F^{\mu \nu}+\frac{1}{2} \sum_{i=1}^{N_{f}} \bar{\lambda}_{i}(\not D+m) \lambda_{i}\right\}=S_{g}+S_{f},
$$

where $F_{\mu \nu}$ is the field strength tensor and the covariant derivative $D_{\mu}$ acts in the adjoint representation. ${ }^{2}$ We consider $\mathrm{SU}\left(N_{c}\right)$ gauge groups and we focus our numerical simulations only on $N_{c}=2$. Two Majorana spinors can be combined to a single Dirac spinor. Hence the counting of Majorana flavors $N_{f}$ corresponds formally up to a factor $1 / 2$ to the Dirac flavour counting used in QCD. The special case of $N_{f}=1 \mathrm{QCD}(\operatorname{adj})$ is $\mathcal{N}=1$ supersymmetric Yang-Mills theory (SYM) and the fermion $\lambda$ is called gluino, the superpartner of the gluon. Supersymmetry is obtained in the massless limit since a finite $m$ breaks supersymmetry softly.

The lattice discretization of QCD(adj) can be chosen in several different ways. We use for the discretization of $S_{g}$ the tree level Symanzik improved gauge action, composed of square and rectangular Wilson loops of size $1 \times 2\left(W_{\mu \nu}^{(2,1)}\right)$ and $1 \times 1\left(W_{\mu \nu}^{(1,1)}\right)$,

$$
S_{g}=-\frac{\beta}{N_{c}}\left(\frac{5}{3} \sum_{x, \mu>\nu} \operatorname{Tr}\left\{W_{\mu \nu}^{(1,1)}(x)\right\}-\frac{1}{12} \sum_{x, \mu>\nu} \operatorname{Tr}\left\{W_{\mu \nu}^{(2,1)}(x)+W_{\nu \mu}^{(1,2)}(x)\right\}\right) .
$$

The discretization of $S_{f}$ is more involved. According to the Nielsen-Ninomiya theorem the implementation of a local Dirac operator on the lattice either leads to additional fermion degrees of freedom (doublers) or requires the breaking of chiral symmetry. In our approach we use a Wilson-Dirac operator which introduces an additional spin-diagonal term to decouple the doubler modes at the cost of an explicit chiral symmetry breaking, see e.g. for a description of doublers and Wilson term [30]. ${ }^{3}$ The fermion part of our lattice action is

$$
S_{f}=\frac{1}{2} \sum_{x y} \bar{\lambda}_{x}\left(D_{w}\right)_{x y} \lambda_{y}
$$

\footnotetext{
${ }^{2} \mathrm{~A}$ summation of colour indices is assumed.

${ }^{3}$ Overlap and domain wall fermions would be an alternative with a more controlled chiral symmetry breaking but their computational cost is quite demanding. Staggered fermions have been used in earlier investigations of $\mathrm{QCD}(\mathrm{adj})$, but they introduce additional degrees of freedom (tastes) and represent effectively theories with larger $N_{f}$ if rooting is not included.
} 
with the Wilson-Dirac operator

$$
\begin{aligned}
\left(D_{w}\right)_{x, a, \alpha ; y, b, \beta}= & \delta_{x y} \delta_{a, b} \delta_{\alpha, \beta}-\kappa \sum_{\mu=1}^{4}\left[\left(1-\gamma_{\mu}\right)_{\alpha, \beta}\left(V_{\mu}(x)\right)_{a b} \delta_{x+\mu, y}\right. \\
& \left.+\left(1+\gamma_{\mu}\right)_{\alpha, \beta}\left(V_{\mu}^{\dagger}(x-\mu)\right)_{a b} \delta_{x-\mu, y}\right]-\frac{\kappa c_{s w}}{2} \delta_{x y} \sigma_{\mu \nu} F^{\mu \nu},
\end{aligned}
$$

where $V_{\mu}(x)$ are the link variables in the adjoint representation and $F_{\mu \nu}$ is the clover plaquette. The hopping parameter $\kappa$ is related to the bare gluino mass $m_{0}$ via $\kappa=1 /\left(2 m_{0}+8\right)$.

We have explored different strategies for the tuning of the gauge coupling $\beta=\frac{2 N_{c}}{g^{2}}$ and of the parameters of the Wilson-Dirac operator. The first natural choice is the same setting as employed in earlier investigations of the bound states of the theory [31]. In these simulations the clover coefficient $c_{s w}$ has been set to zero and one level of stout smearing has been applied on the links $V_{\mu}(x)$ of the Dirac operator. In this framework $\beta=1.75$ is good compromise between the simulation cost and the control of lattice supersymmetry breaking $[31,32]$. In an alternative setup, we have also simulated the theory using unsmeared links and $c_{s w}=1$, such that the leading order chiral symmetry breaking effects are removed from on-shell quantities [33]. Different lattice actions provide the same information about the phase structure of the theory up to lattice discretization errors, enabling us to check the reliability and the consistency of our simulations.

The integration of each Majorana fermion field yields the Pfaffian $\operatorname{Pf}\left(C D_{w}\right)$, where $C$ is the charge conjugation matrix. The Pfaffian is real but not necessarily positive on lattice. In the continuum limit, the Pfaffian is real and positive. A sign problem appears in the Wilson formulation for odd $N_{f}$ in regions where the mass of the "adjoint pion", the lightest gluino-ball, is small and lattice artefacts are dominant. The sign problem vanishes in the continuum limit. Based on our previous experience of lattice simulations of $\mathcal{N}=1 \mathrm{SYM}$, we already know in the bare parameter space where the contribution from negative Pfaffian configurations is negligible. A positive Pfaffian is assumed in the following sections.

In numerical simulations the lattice extend is finite in all directions. Periodic boundary conditions are always applied in the three spacial directions. Our aim is the investigation of the compactified theory on a torus. Hence the extend in the spacial directions is assumed to be large enough to emulate the infinite volume limit and the temporal direction is compactfied:

$$
\underbrace{T^{3}}_{\text {large }} \times S^{1} \approx \mathbb{R}^{3} \times S^{1}
$$

If fermions fields fulfill anti-periodic boundary conditions in the $S^{1}$ direction, the theory on the $T^{3} \times S^{1}$ torus emulates the thermal partition function of a quantum field theory in a box. If periodic boundary conditions are applied to fermion fields on $S^{1}$ direction, the theory on $T^{3} \times S^{1}$ corresponds to the twisted partition function (1.1) with no thermal interpretation. The latter setup is useful to realize the notion of adiabatic continuity.

Lattice simulations have to challenge discretization and finite volume effects. Finally, numerical instabilities of the Rational Hybrid Monte Carlo algorithm forbid simulations at very small gluino masses. Hence SYM can be simulated only with a non-vanishing soft 
supersymmetry breaking mass term. The continuum limit and the massless limit must be extrapolated from the numerical data.

\section{Order parameters for the phase diagram of adjoint QCD}

The lattice action is invariant under center symmetry transformations, corresponding to the multiplication of the gauge links in time direction on a given time-slice with a discrete phase rotation $\exp \left(i \phi_{n}\right)$ with $\phi_{n}=2 \pi n / N_{c}$. The deconfinement transition is identified with the spontaneous breaking of this symmetry at high temperatures. The quark fields in QCD, which are fermions in the fundamental representation, break center symmetry explicitly if one imposes periodic or anti-periodic boundary conditions. This symmetry is, however, preserved for fermions in the adjoint representation.

The Polyakov loop is the order parameter of the deconfinement phase transition. It is the path ordered product of the links in the fundamental representation along a line which wraps in the compact direction

$$
P_{L}=\frac{1}{N_{c} V_{3}} \sum_{\vec{x}} \operatorname{Tr} W^{\left(N_{t}\right)}(\vec{x})=\frac{1}{N_{c} V_{3}} \sum_{\vec{x}} \operatorname{Tr}\left\{\prod_{t=1}^{N_{t}} U_{4}(\vec{x}, t)\right\},
$$

where $V_{3}$ denotes the three-dimensional lattice volume of the non-compactified directions. The confined and deconfined phases are distinguished by a zero or non-zero expectation value of $P_{L}$. We consider in addition the Polyakov loop in adjoint representation $P_{L}^{A}$, the path ordered product of links in the adjoint representation. The adjoint Polyakov loop is not an order parameter of the deconfinement transition due to the screening of the adjoint color charge. More generally arbitrary windings $n$ of the Polyakov loop can be considered

$$
P_{L}^{(n)}=\frac{1}{N_{c} V_{3}} \sum_{\vec{x}} \operatorname{Tr}\left[\left(W^{\left(N_{t}\right)}(\vec{x})\right)^{n}\right],
$$

which is equivalent to consider loops in all representations. The eigenvalues of the Polyakov line $W^{\left(N_{t}\right)}$ play an important role in the discussions of the perturbative effective action in section 4.

In the massless limit, classical SYM has an additional U(1) chiral symmetry $\lambda \rightarrow \exp \left\{-\mathrm{i} \theta \gamma_{5}\right\} \lambda$. This is not a symmetry in quantum theory due to the global Adler-BellJackiw anomaly. The genuine symmetry of the quantum theory is $\mathbb{Z}_{2 N_{c}}$ in case of the gauge group $\mathrm{SU}\left(N_{c}\right)$. The discrete symmetry is broken spontaneously to $\mathbb{Z}_{2}$, and the theory has $N_{c}$ isolated discrete vacua. The chiral condensate is the order parameter for this transition. It is defined as the derivative of the logarithm of the partition function with respect to the fermion mass, equal to the expectation value of the quark bilinear $\langle\bar{\lambda} \lambda\rangle$. This operator is multiplicatively and additively renormalized in the Wilson formulation of lattice fermions. In case of $N_{f}>1$ more general condensates like $\left\langle\operatorname{det}\left(\lambda_{i} \lambda_{j}\right)\right\rangle$ are possible [4] which we have not considered in our present work.

A susceptibility can be defined for the order parameters $O$ as

$$
\chi_{O}=V\left(\left\langle O^{2}\right\rangle-\langle O\rangle^{2}\right),
$$


where $V$ is the three-dimensional volume for the Polyakov loop or the four-dimensional volume for the chiral condensate. The phase transition can be identified by the peak of the susceptibility and the divergence of this peak in the infinite volume limit. The susceptibility is subject to rather large finite volume corrections. The Binder cumulant,

$$
B_{4}(O)=1-\frac{1}{3} \frac{\left\langle O^{4}\right\rangle}{\left\langle O^{2}\right\rangle^{2}},
$$

provides a signal with smaller finite size corrections. The deconfinement transition is expected to be in the three-dimensional Ising universality class [34], and the $B_{4}$ value at the transition should be 0.46548(5) [35], while in the confined and deconfined phase it is equal to zero and $2 / 3$ respectively.

In previous numerical simulations we have determined the deconfinement and chiral transitions in SYM at finite temperature. With thermal boundary conditions, the temperature corresponds the inverse of the compactification radius $T=1 / R$. We have been able to observe a significantly different behaviour in the case of periodic boundary conditions for fermion fields in [29], by exploring the phase diagram in the space of the bare lattice parameters $\beta$ and $\kappa$ at fixed $N_{t}$. The deconfinement transition line does not intersect the critical line corresponding to the zero renormalized gluino mass. The clear separation of the two lines is already a signal of continuity, but the control of lattice artefacts and the interpretation of the phase diagram in terms of renormalized physical quantities is difficult. We follow a different approach in the present work. We keep fixed bare parameters, therefore the lattice spacing $a$ and the gluino mass are constant, and the length of the compactification radius is changed by changing the number of lattice points $N_{t}=R / a$ in the temporal (compactified) direction.

\section{The perturbative effective potential for the Polyakov loop on the lattice}

The confined and deconfined phases as a function of $R, m$ and $N_{f}$ can be inferred from the effective potential of the Polyakov line that can be calculated straightforwardly in the one-loop approximation. The effective potential is usually identified with the free energy density at a constant background gauge field $G_{\mu}$, which is at one loop level determined from the quadratic fluctuations. The background is chosen such that the link in time direction $G_{4}$ is diagonal and all other links are set to the identity. In the case of $\mathrm{SU}(2)$ we have

$$
G_{4}=\left(\begin{array}{cc}
e^{i \phi / N_{t}} & 0 \\
0 & e^{-i \phi / N_{t}}
\end{array}\right)
$$

and Polyakov loop then reads

$$
P_{L}=\frac{1}{2} \operatorname{Tr}\left\{\prod_{t=1}^{N_{t}} G_{4}\right\}=\frac{1}{2} \operatorname{Tr}\left(\begin{array}{cc}
e^{i \phi} & 0 \\
0 & e^{-i \phi}
\end{array}\right)=\cos (\phi) .
$$

Confinement (center-stability) is realized if the minima of the effective potential for the Polyakov line phase (holonomy) $\phi$ is located at $\phi=\pi / 2 \bmod \pi$. In the deconfined phase 
there is a minimum at $\phi=0 \bmod \pi$. For a general $\mathrm{SU}\left(N_{c}\right)$ gauge group, the background configuration is

$$
G_{4}=\operatorname{diag}\left[e^{i \phi_{1} / N_{t}}, e^{i \phi_{2} / N_{t}}, \ldots, e^{i \phi_{N_{c}} / N_{t}}\right],
$$

where $\phi_{N_{c}}=-\sum_{i=1}^{N_{c}-1} \phi_{i}$ and

$$
P_{L}=\frac{1}{N_{c}} \operatorname{Tr}\left\{\prod_{t=1}^{N_{t}} G_{4}\right\}=\frac{1}{N_{c}} \operatorname{Tr}\left(\begin{array}{llll}
e^{i \phi_{1}} & & & \\
& e^{i \phi_{2}} & & \\
& & \ddots & \\
& & & e^{i \phi_{N_{c}}}
\end{array}\right)
$$

The effective potential of $\mathrm{QCD}(\mathrm{adj})$ in the one loop approximation is the sum of gluons, ghost, and fermion contributions. On the lattice the gauge links with the fluctuating field $A_{\mu}$ around the constant background $G_{\mu}$ are represented as

$$
U_{\mu}(x)=G_{\mu} \exp \left\{i g A_{\mu}(x)\right\},
$$

and the gluon action is expanded in a power series of $g$. The kinetic part of the gluon Lagrangian is

$$
\mathcal{L}_{g l}=\frac{1}{2} \operatorname{Tr}\left\{D_{\mu}^{+} A_{\nu}(x) D_{\mu}^{+} A_{\nu}(x)\right\}
$$

where the gauge fixing term

$$
S_{g f}=\sum_{x, \mu \nu} \operatorname{Tr}\left\{D_{\mu}^{-} A_{\mu}(x) D_{\nu}^{-} A_{\nu}(x)\right\}
$$

has been added to the action to fix the gauge of the field $A_{\mu}(x)$ while preserving gauge invariance with respect to background field $G_{\mu}$. Only the standard Wilson plaquette action part is relevant in this computation. $D^{+}$and $D^{-}$denote the forward and backward lattice covariant derivatives

$$
\begin{aligned}
& D_{\mu}^{+}=\frac{1}{a}\left(G_{\mu}^{\dagger}(x) A_{\mu}(x+\mu) G_{\mu}(x)-A_{\mu}(x)\right) \\
& D_{\mu}^{-}=\frac{1}{a}\left(A_{\mu}(x)-G_{\mu}^{\dagger}(x-\mu) A_{\mu}(x-\mu) G_{\mu}(x-\mu)\right) .
\end{aligned}
$$

The kinetic part of the ghost field $\eta$ is similarly

$$
\mathcal{L}_{g h}=\frac{1}{2} \operatorname{Tr}\left\{D_{\mu}^{+} \eta(x) D_{\mu}^{+} \eta(x)\right\}
$$

the last contribution comes from the Wilson fermion action $\left(m_{0}=a m\right)$

$$
\mathcal{L}_{f}=\frac{1}{2} \sum_{f=1}^{N_{f}} \bar{\lambda}^{f}(x)\left\{\gamma_{\mu} \frac{D_{\mu}^{+}+D_{\mu}^{-}}{2}+\operatorname{ar} D_{\mu}^{+} D_{\mu}^{-}+m\right\} \lambda^{f}(x) .
$$

The action is quadratic in fluctuating fields $A_{\mu}, \eta$ and $\lambda$ in the one-loop approximation, therefore these fields can be integrated out leading to

$$
\begin{aligned}
V_{3} V_{\text {eff }} & =\left(\frac{4}{2}-1\right) \log \operatorname{det}\left(D_{\mu}^{-} D_{\mu}^{+}\right)-\frac{N_{f}}{2} \log \operatorname{det}\left(\gamma_{\mu} \frac{D_{\mu}^{+}+D_{\mu}^{-}}{2}+\operatorname{ar} D_{\mu}^{+} D_{\mu}^{-}+m\right) \\
& =\log \operatorname{det}\left(D_{\mu}^{-} D_{\mu}^{+}\right)-N_{f} \log \operatorname{det}\left(\frac{1}{4}\left(D_{\mu}^{+}+D_{\mu}^{-}\right)^{2}+\left(\operatorname{ar} D_{\mu}^{+} D_{\mu}^{-}+m\right)^{2}\right) .
\end{aligned}
$$


Each boson field contributes to $V_{\text {eff }}$ with a prefactor $+\frac{1}{2}$, and each fermion field with a prefactor -1 . The first term comes from the gauge part of the action, with a prefactor which counts four boson fields $A_{\mu}$ minus the fermion ghost field $\eta$. The last term is the contribution from the Majorana fermions with an additional factor $\frac{1}{2}$ due to the fact that integrating our fermion induce a Pfaffian.

The effective potential (4.12) shows clearly how a mismatch between fermion and boson contributions is introduced by the lattice discretization. The Wilson fermions have a different derivative operator and an additional momentum dependent mass term in order to remove the doubling modes. In the continuum, a more compact expression for $V_{\text {eff }}$ is obtained in the massless case. It can be recovered in the naive $a \rightarrow 0$ limit of (4.12)

$$
V_{3} V_{\text {eff }}=\left(1-N_{f}\right) \log \operatorname{det}\left(D^{2}\right)
$$

since $\log \operatorname{det}(\not D)=\frac{1}{2} \log \operatorname{det}(\not D D D)=\frac{1}{2} \log \operatorname{det}\left(D^{2}\right)$. In this limit the difference between the boson and fermion derivative operators disappears. Gluon and adjoint fermion fields have an opposite contribution to the one-loop effective potential of the Polyakov loop which cancel exactly in the continuum if $N_{f}=1$, i. e. in the case of the $\mathcal{N}=1$ SYM. Supersymmetry ensures that this result holds to all orders of perturbation theory. In continuum $\mathcal{N}=1$ SYM theory, center stability is driven by pure non-perturbative effects [19]. For $N_{f}>1$ continuum QCD (adj), the center-stability is a one-loop perturbative effect. However, the supersymmetry between gluons and gluinos of SYM is violated on the lattice, and $V_{\text {eff }}$ differs from zero for non-zero lattice spacings. This mismatch will play an interesting role in section 5.1 in order to explain the difference of the lattice and continuum phase diagram.

The effective potential for the Polyakov line holonomies $\phi_{a}$ (or their differences $\phi_{a b}=$ $\left.\phi_{a}-\phi_{b}\right)$ can be further simplified in momentum space [36, 37]. Assuming an infinite spacial extend of the lattice, an $\infty^{3} \times N_{t}$ site lattice, and neglecting holonomy independent contributions, we obtain the following representation

$$
\begin{aligned}
V_{\mathrm{eff}}\left(\left\{\phi_{a}\right\}\right)= & \sum_{a \neq b} V_{\mathrm{eff}}\left(\phi_{a b}\right)=\sum_{k=0}^{N_{t}-1} \sum_{a \neq b} \int_{-\pi}^{\pi}\left(\frac{d p}{2 \pi}\right)^{3} \\
& \left\{\log \left[\hat{p}^{2}+4 \sin ^{2}\left(\frac{\phi_{a b}+2 \pi k}{2 N_{t}}\right)\right]-N_{f} \log \left[\hat{\hat{p}}^{2}+\sin ^{2}\left(\frac{\phi_{a b}+2 \pi k}{N_{t}}\right)\right.\right. \\
& \left.\left.+\left(m_{0}+\frac{r}{2}\left[\hat{p}^{2}+4 \sin ^{2}\left(\frac{\phi_{a b}+2 \pi k}{2 N_{t}}\right)\right]\right)^{2}\right]\right\}
\end{aligned}
$$

where the lattice momenta $\hat{p}$ and $\hat{\hat{p}}$ are defined as

$$
\hat{p}^{2}=\sum_{i=1}^{3} 4 \sin ^{2}\left(\frac{p_{i}}{2}\right) \quad \text { and } \quad \hat{\hat{p}}^{2}=\sum_{i=1}^{3} \sin ^{2} p_{i} .
$$

Consider first $r=0$ and $m_{0}=0$, and set the holonomy field to zero in (4.14). The difference between boson and fermion derivatives on the lattice is essentially reflected in $\hat{p}^{2}$ vs. $\hat{\hat{p}}^{2}$. In fact, for bosons, the inverse propagator vanishes only at the origin of the Brillouin 
zone, as in the continuum case. However, for fermions, the inverse propagator is zero at all sixteen corners of Brillouin zone, $(0,0,0,0),( \pm \pi, 0,0,0), \ldots,( \pm \pi, \pm \pi, \pm \pi, \pm \pi)$. The corners except the origin are referred to as doublers, and undesired from the continuum point of view. On the lattice, the Wilson term proportional to $r$ leads to a momentum dependent mass that removes the doublers in the continuum limit. The Wilson term lifts the doublers by making the modes at $p_{\mu} \sim \pi$ acquire a mass of the order of cut-off, i.e. proportional to the inverse lattice spacing. However, the Wilson term explicitly breaks chiral symmetry, as a consequence the fermion mass is both additively and multiplicatively renormalized.

The difference of the fermion determinants of the free theory is a shift of the modes in the compact or temporal direction by $\phi_{a b}$. This result generalizes to any kind of fermion operator that can be implemented on the lattice: the effective potential is easily derived from a shifted momentum space representation. The shifts for the adjoint representation are $\frac{\phi_{a b}}{N_{t}}$, for the fundamental one they would be $\frac{\phi_{a}}{N_{t}}$. In the case of $\mathrm{SU}(2)$ there is only one shift for the adjoint representation: $\phi_{12}=-\phi_{21}=2 \phi$.

The effective potential for $\phi_{a b}$ can be translated into an effective potential of different windings of the Polyakov loop. Again up to constant expressions, the quadratic potential

$$
V_{\text {eff }}\left(\left\{\phi_{a}\right\}\right)=\sum_{n=1}^{\infty} \sum_{a \neq b} V_{\text {eff }}^{(n)} e^{i r \phi_{a b}}=N_{c}^{2} \sum_{n=1}^{\infty} V_{\text {eff }}^{(n)}\left|P_{L}^{(n)}\right|^{2}+\text { const }
$$

is obtained. The coefficients $V_{\text {eff }}^{(n)}$ are the Fourier transform of $V_{\text {eff }}\left(\phi_{a b}\right)$ with respect to $\phi_{a b}$

$$
V_{\mathrm{eff}}^{(n)}=\int_{0}^{2 \pi} \frac{d \phi_{a b}}{2 \pi} e^{-i r \phi_{a b}} V_{\mathrm{eff}}\left(\phi_{a b}\right)
$$

After some algebra, the following expression is obtained for the coefficients $V_{\text {eff }}^{(n)}$

$$
\begin{aligned}
V_{\mathrm{eff}}^{(n)}= & N_{t} \int_{-\pi}^{\pi} \frac{d \omega}{2 \pi} e^{i r N_{t} \omega} \int_{-\pi}^{\pi}\left(\frac{d p}{2 \pi}\right)^{3} \\
& \log \left(\frac{\left[\hat{p}^{2}+4 \sin ^{2}\left(\frac{\omega}{2}\right)\right]}{\left[\hat{\hat{p}}^{2}+\sin ^{2}(\omega)+\left(m_{0}+\frac{r}{2}\left(\hat{p}^{2}+4 \sin ^{2}\left(\frac{\omega}{2}\right)\right)\right)^{2}\right]^{N_{f}}}\right) .
\end{aligned}
$$

If the coefficients $V_{\text {eff }}^{(n)}$ are positive for $n \leq\left\lfloor\frac{N_{c}}{2}\right\rfloor, \mathbb{Z}_{N_{c}}$ center-symmetry is preserved and the minimum of the potential is at $P_{L}^{(n)}=0$ for $n \neq 0 \bmod N_{c}$; i.e, the expectation value of the Polyakov loops with winding number $n \neq 0 \bmod N_{c}$ is zero.

If some of the $V_{\text {eff }}^{(n)}$ are negative for $n \leq\left\lfloor\frac{N_{c}}{2}\right\rfloor$, a corresponding subset of Polyakov loops will develop non-zero expectation values. In fact, if $V_{\text {eff }}^{(n)}<0$ for $n=1$, then so are all higher $n$. In this case, $\mathbb{Z}_{N_{c}}$ center-symmetry is completely broken. If $V_{\text {eff }}^{(n)}<0$ for $1 \leq n \leq k \leq\left\lfloor\frac{N_{c}}{2}\right\rfloor$, then the center symmetry may break to different discrete subgroups that can be easily determined.

Note that an increase of $\frac{1}{V_{\text {eff }}^{(n)}}$ indicates also an increasing susceptibility of the Polyakov line with winding number $n$, providing a signal for the effective potential investigated in section 6 . 


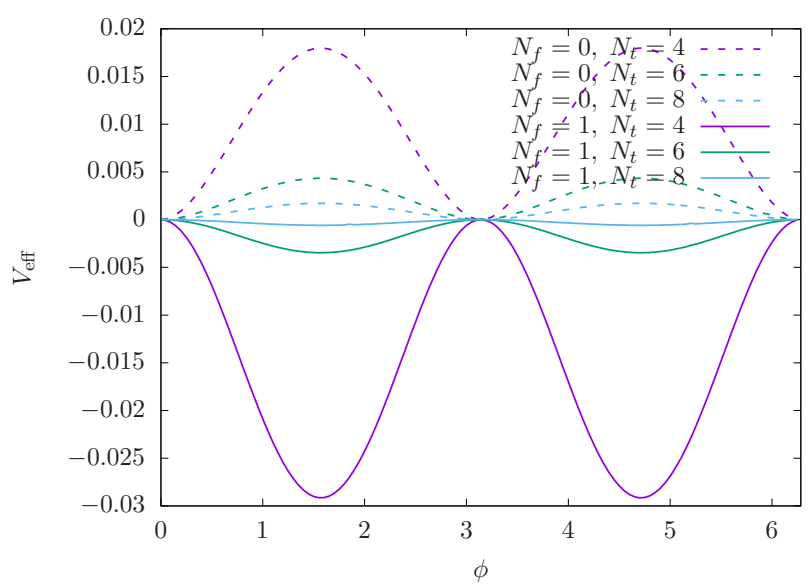

Figure 1. One-loop effective potential for the Polyakov loop phase $\phi$ in SU(2) SYM on the lattice for different $N_{t}$ at $m_{0}=0$ comparing pure gauge $\left(N_{f}=0\right)$ and SYM $\left(N_{f}=1\right)$. The SYM effective potential is different from zero due to lattice artefacts. It is confining and the expected flat behavior is reached only asymptotically at large $N_{t}$. The potentials are normalized to zero at $\phi=0$ in this plot.

We have computed the one-loop effective potential of SU(2) SYM on the lattice (4.12) or equivalently (4.14) for several values of $N_{t}$. Clearly, unlike the continuum supersymmetric theory, for which the effective potential is zero to all orders in the perturbative expansion due to supersymmetry, the one-loop potential in the lattice formulation (4.12), (4.14) is actually non-vanishing,

$$
\begin{aligned}
V_{\text {eff, continuum }}\left(\left\{\phi_{a}\right\}\right) & =0, \\
V_{\text {eff, lattice }}\left(\left\{\phi_{a}\right\}\right) & =N_{c}^{2} \sum_{n=1}^{\infty} V_{\text {eff }}^{(n)}\left|P_{L}^{(n)}\right|^{2} \text { with } V_{\text {eff }}^{(n)}>0 \text { for } n \leq\left\lfloor\frac{N_{c}}{2}\right\rfloor .
\end{aligned}
$$

This is hardly surprising because the lattice formulation based on Wilson fermions does not respect supersymmetry, and as such, we indeed expect a potential to be induced at one-loop order. The correct continuum limit is approached as $R / a$ goes to infinity, which means the lattice spacing goes to zero at fixed physical $R$.

Remarkably, Wilson fermions with Wilson parameter $r=1$, even when the effect of doublers is maximally lifted, have a stronger confining effect than continuum fermions, see figure 1, and lead to center stability even at one-loop level. In this respect, lattice SYM is similar to the $N_{f}>1$ continuum QCD(adj) [38]. The one-loop prediction is a center-symmetric (confined) phase in the small $R$ regime at a given fixed lattice spacing and $m_{0}=0$. In the small $R / a$ regime, the dynamics probes higher scales towards the cutoff $1 / a$. At these scales the doubling modes that are lifted with masses of the order of the cutoff are still relevant and have a confining effect.

If the mass is increased, a phase transition to a deconfined phase occurs [38], calculable both in lattice perturbation theory and by continuum methods, as discussed in section 5 . This phase is connected to the deconfined phase of pure Yang-Mills theory. At $N_{c}>2$ 
the deconfinement transition is replaced by a transition to several intermediate phases with partially broken gauge symmetry, corresponding to the Higgs phases in the Hosotani mechanism [39]. The deconfined phase connected to the pure Yang-Mills case is reached increasing $R$ after crossing these additional phases.

\subsection{Abelian vs. non-Abelian confinement regimes: first pass}

In the following we discuss in more detail the particular features of the confinement mechanisms at small radius that arise in $\mathrm{QCD}(\operatorname{adj})$ at non-zero lattice spacing in the $N_{f}=1$ case and also for $N_{f}>1$.

The unbroken center symmetry at any $R \in[0, \infty)$ does not have a unique implication for the Polyakov loop eigenvalues. Originally, Polyakov thought that the phases of the loop would have completely random fluctuations as origin of the a vanishing expectation value for the Polyakov line operator in the confined phase. However, from the analysis of the confining perturbative effective potential we obtain two different pictures. Indeed, both at small and large- $R,\left\langle P_{L}\right\rangle=0$, but at small- $R$ the (untraced) Polyakov line is essentially $\left(\begin{array}{cc}e^{i \frac{\pi}{2}} & 0 \\ 0 & e^{-i \frac{\pi}{2}}\end{array}\right)$ with small-fluctuations around it. We refer to this regime as Abelian confinement regime, where the theory dynamically abelianized due to the induced potential (4.12), (4.14). ${ }^{4}$

At large- $R$, the theory is strongly coupled at the compactification scale and the fluctuations of the Polyakov loop eigenvalues are random as it can be seen from simulation results presented in section 6.3. We refer to this regime as non-Abelian confinement regime.

Some intuition for the large- $R$ case may be gained from strong coupling lattice perturbation theory. However, note that the strong coupling here refers to the bare lattice coupling at the cut-off. The strong coupling regime of lattice theory is not necessarily continuously connected to the continuum physics, yet the strong coupling expansion provide some insights to understand lattice results. In particular, at infinite bare coupling, $\beta=0$, we can ignore the action and the first term in the strong coupling expansion is the reduced Haar measure with an effective potential for the Polyakov line eigenvalues

$$
V_{\text {Haar }}=-\frac{1}{2} \sum_{a \neq b} \log \left[\sin ^{2}\left(\frac{\phi_{a b}}{2}\right)\right] \text {. }
$$

The reduced Haar measure will always drive the theory to the confined phase. Since we are discussing the continuum physics that arises from bare weak coupling (as per asymptotic freedom) of lattice field theory, we will not discuss strong coupling expansion. Nevertheless

\footnotetext{
${ }^{4}$ Dynamical abelianization is interesting on its own right, and the class of theories we are examining exhibits some differences from other calculable confining QFTs. In Georgi-Glashow model in threedimensions [1, 40,41] and Seiberg-Witten theory in four-dimensions [42], the long distance theory abelianizes due to a tree-level (classical) potential. In $\mathrm{QCD}(\mathrm{adj})$, at tree level, there is no potential for Polyakov loop. It is dynamically induced at one loop level, and leads to abelianization of the long distance theory [4]. Dynamical abelianization should also not be confused with 't Hooft's maximal abelian gauge proposal [43], which enforces abelianization by hand as a gauge choice. Needless to say, this is done in the QFT which becomes strongly coupled at large-distance and in particular, is not semi-classically calculable.
} 
the Haar measure has to be considered as an important contribution in the lattice simulations. In our numerical studies, we use the distribution of the per-site Polyakov loop phase $\phi$ normalized with respect to the Haar measure for different $R$ to gain a sense of distinction between Abelian and non-Abelian confinement regimes.

The picture that emerges by the examination of the thermal and graded partition functions corresponding to thermal and periodic compactification with analytic (lattice perturbation theory at weak coupling domain) and numerical (both at weak and strong coupling domain) methods is as follows: at low temperatures or large-circle size $R$ where the theory becomes strongly coupled, there are rather unconstrained fluctuations of the $\phi_{a b}$, which leads to a vanishing Polyakov line expectation value. At higher temperatures, or small circles, the weak coupling at the scale of the circle size admits a calculable potential for the Polyakov loop, which may lead to either to a confined or deconfined vacuum depending on the details. The Polyakov line expectation value is in this case determined by the minimum of the effective potential of $\phi_{a b}$ and the randomness of the fluctuations becomes suppressed, regardless of whether the theory is confined or deconfined. However, the suppression of the randomness of fluctuations will happen in different ways: in the thermal deconfined phase, there is a dominance of the $\phi=0$ center-broken configuration, in the periodic confined phase, a dominance of the $\phi=\frac{\pi}{2}$ center-symmetric configuration. The center-symmetric suppression of the randomness of fluctuations is a signal of the dynamical abelianization according to the Abelian confinement picture. The confinement that takes place at large-circle (strong renormalized coupling) is referred to as non-Abelian confinement picture. In the present work, we provide evidence for the continuity between the two.

It is worth mentioning that historically well-known examples of Abelian confinement mechanisms such as the three-dimensional Polyakov model [1] and the softly broken Seiberg-Witten theory in four dimensions [42]. These theories have a property which is unlike pure Yang-Mills theory. In pure Yang-Mills theory, $k$-string tensions are classified by $N_{c^{-}}$-ality. There is only one-type of string between a quark with $N_{c^{-}}$ality $k$ and its anti-quark with tension $T_{k}$. Consider for example the 1-string tension $T_{1}$. However, in the above mentioned theories, since the gauge group is $\mathrm{U}(1)^{N_{c}-1}$ and since the Weyl symmetry permuting gauge group factors are spontaneously broken, $T_{1}$ is replaced by $N_{c}-1$ fundamental strings, $T_{1, j}$ as shown in [44]. This is not the case in $\mathrm{QCD}(\operatorname{adj})$ as well as deformed Yang-Mills theory, where the $\mathbb{Z}_{N_{c}}$ sub-group of the Weyl group remains intact in the Abelian confinement regime. Consequently, there is again only one-type of fundamental string, see [45] for details.

\subsection{Different Polyakov line effective actions}

A deep understanding of the different non-perturbative definitions of the Polyakov line effective action is required to link lattice results to the one-loop perturbative calculations. There is a difference between the effective action for the Polyakov loop $P_{L}$ and the one of $\phi_{a}$. These two effective actions are related for a constant background by the Fourier representation of eq. (4.16), but there is no general identification between the expectation values $\left\langle P_{L}\right\rangle$ and $\left\langle\phi_{a}\right\rangle$. We won't enter the discussion about the convexity of the effective action since it is not relevant for our discussions, see [46] for further details. 
The relevant counterpart of the effective action that is easily accessible on the lattice is the constraint effective potential, see [46-48] for a discussion. Up to an overall constant, it is the logarithm of the probability density of the observable $O$

$$
V_{\mathrm{c}}(\Phi)=-\frac{1}{V} \log \left[\int \mathcal{D} \theta \delta(\Phi-O(\theta)) e^{-S[\theta]}\right]=-\log \left[P_{\mathrm{pdf}, O}(\Phi)\right]+\text { constant } .
$$

The integration variable $\theta$ represents all gauge and fermion fields. The probability density $P_{\text {pdf }, O}(\Phi)$ can be numerically approximated by the histogram of $O$ obtained from the Monte-Carlo data. The most relevant information can be already extracted from the lowest moments of the distribution. A crucial role is played by the second moment which is equivalent to the susceptibility of eq. (3.3). The susceptibility is the inverse of the coefficient in front of the quadratic term in the effective potential.

The definition (4.21) shows that the identification of the phase to the Polyakov line effective potential in eq. (4.16) is unambiguous only in the constant field limit. In the general case, the Polyakov line eigenvalues and the phases are space-dependent. The $\phi_{a}$ in the effective potential can be identified with the volume averaged phase factors or the phases of the volume averaged Wilson lines. In both cases the direct application of the perturbative formulas is not possible since either the volume averaged loop can not be determined from the volume average phases, or the volume averaged Wilson line is not a group element. A qualitative agreement with the potential of eq. (4.16) is still expected, especially in the strong and weak coupling limit where the fluctuations of the phase factors are suppressed.

A constraint effective potential can also be defined from the per-site distribution of the observable. This distribution can be determined with a better accuracy due to the additional volume factor in the statistical sampling. The per-site distribution is significantly different from the distribution of the volume averaged observable. Smoothing techniques of the field configuration can be applied in order to match the two distributions, but still the infinite volume limit is not well defined for the per-site distribution. In the strong coupling limit the per-site constraint effective potential agrees with the constraint effective potential of the Polyakov line. This leads to the several different definitions of the Polyakov line effective potential mentioned in the literature: the Polyakov line effective potential, the per-site Polyakov line effective potential and the same for the effective potential of $\phi_{a}$.

The per-site distribution has been investigated in particular to confirm the non-Abelian confinement. As explained above, the contributions of the Haar measure have to be factorized out in order make contact to this view of the confinement mechanism. Such an analysis has been done in [39] for the case of $\mathrm{SU}(3) \mathrm{QCD}(\mathrm{adj})$ with $N_{f}>1$ and we perform a similar analysis here for SU(2) SYM. In addition, we discuss the susceptibility in order to get information about the form of the effective action from the numerical simulations.

\section{The phase diagram on the continuum and lattice}

Below, we review the phase diagram of $\mathcal{N}=1 \mathrm{SYM}$ and $\mathrm{QCD}(\mathrm{adj})$ with periodic boundary condition on $\mathbb{R}^{3} \times S^{1}$. Part of these phase diagrams are calculable by weak coupling methods 


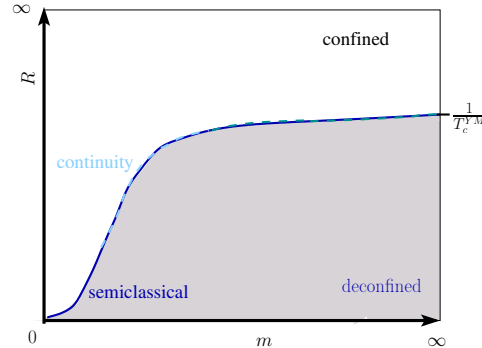

(a) SYM continuum.

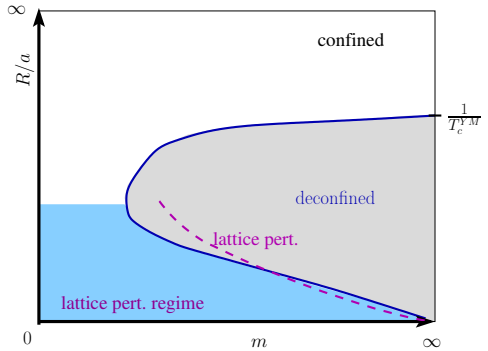

(b) SYM lattice.

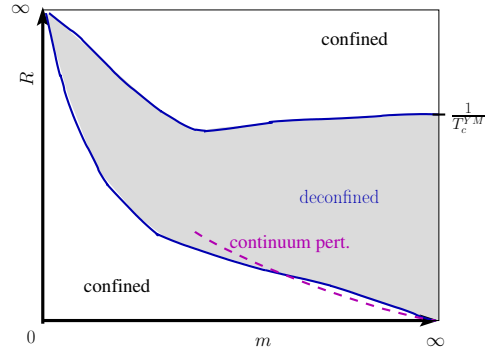

(c) $N_{f}^{*}<N_{f}<N_{f}^{\text {a.f. }}$.

Figure 2. A sketch of the expected phase diagrams in $N_{f} \mathrm{QCD}$ (adj). Figure (a) is the continuum case in SYM with the semiclassical predictions valid on the small $(R, m)$ corner, the well established numerical results at large- $m$ (where semi-classic does not apply) and the conjectured continuity of the transition line. Figure (b) is the phase diagram of SYM on the lattice at a fixed lattice spacing. At small $R / a$ regime the transition line approaches lattice perturbation theory. The "lattice pert. regime" corresponds to a region where the coupling stays small at the scale of compactification. There are crucial discretization effects in this regime. In this regime the theory behaves similar to continuum QCD(adj) with larger $N_{f}$. (Figure (c)) If $N_{f}^{*}<N_{f}<N_{f}^{\text {a.f. }}$ becomes larger than a certain critical value dictated by lower end of conformal window and smaller than asymptotic freedom bound, the theory on decompactification limit is a CFT. However, upon compactification (with periodic boundary conditions), center is stable and confinement sets in. Continuum one-loop perturbative analysis can be used to show that $R_{c} m=3.484$ for $N_{f}=5$ (lower transition line). The upper transition line is non-perturbative and not calculable.

involving a combination of perturbative one-loop effective potential for Wilson line and non-perturbative semi-classical methods.

Next, we describe the calculable critical compactification radius $R_{c}$ as a function of $N_{f}$ and $m_{0}$ by using the one-loop effective potential on the lattice. We also briefly review the part of the phase diagram that is accessible by lattice techniques, but not via weak coupling methods.

Continuum, $\mathbf{N}_{\mathbf{f}}=1 \mathbf{Q C D}(\mathbf{a d j})$ : the phase diagram of $\mathcal{N}=1 \mathrm{SYM}$ in the continuum is shown in figure $2(\mathrm{a})$. The small $(m, R)$ corner of this phase diagram is semi-classically calculable and exhibits a center-symmetry changing phase transition due to asymptotic freedom and unbroken center symmetry in the chiral limit $[19,38]$. In the large- $m$ regime, the theory approaches pure Yang-Mills theory as fermions decouple. This regime is not semi-classically calculable, but from lattice simulations, it is known that a transition exists and it is natural to expect that the transition line extrapolates from small $m$ to large- $m$ regime. In this picture, there is only one transition line from confined to deconfined phase. The theory is non-perturbatively confined for all $m<m_{c}$ or $R>R_{c}$ in the calculable limit with a transition boundary at [19]

$$
R_{c}=\Lambda^{-1} \sqrt{\frac{m}{8 \Lambda}} \text { for } N_{f}=1
$$

where $\Lambda$ is the strong scale. 
There are two conjectural continuities in the phase diagram shown in figure 2(a) corresponding to the twisted partition function (1.1):

- Adiabatic continuity between the small- $R$ weak coupling confined phase and large- $R$ regime where the theory becomes strongly coupled at large distances.

- The continuity of semiclassical (weak coupling) and the strong coupling phase transition.

There is a sufficiently strong reason to believe continuity if the fermion mass is zero. (1.1) reduces to the supersymmetric Witten index [18] at $m=0$,

$$
I_{W}=\tilde{Z}(R, m=0)=\operatorname{tr}\left[e^{-R \hat{H}(0)}(-1)^{F}\right]=N_{c} \quad \text { for } \mathrm{SU}\left(N_{c}\right),
$$

which counts the difference of bosonic and fermionic ground states. At fixed small- $m$, for $R<R_{c}$, one can prove the stability of the confined phase, and also prove the existence of center symmetry breaking for $R<R_{c}$ [19]. The main aim of the present work is to find a non-perturbative evidence for the above adiabatic continuity conjectures.

$\mathbf{1}<\boldsymbol{N}_{f}<\boldsymbol{N}_{f}^{\text {a.f. }} \mathbf{Q C D}(\mathbf{a d j})$ : let us first recall the conjectured phase diagram of $\mathrm{QCD}(\operatorname{adj})$ in the continuum. Let $N_{f}^{*}$ denote the putative number of flavors below which the theory is confining and above which it exhibits IR-conformality on $\mathbb{R}^{4}$. For $N_{f} \geq N_{f}^{a . f}$. even asymptotic freedom is lost.

The expected phase diagram for $1<N_{f}<N_{f}^{*}$ is the same as shown in figure 2(b) and for $N_{f}^{*}<N_{f}<N_{f}^{a . f .}$, it is shown in figure 2(c). In both of these diagrams, the upper transition lines are incalculable by weak coupling methods but the lower lines are calculable. The one-loop potential is of the form:

$$
V^{\text {pert. }}(\phi)=N^{2} \sum_{n=1}^{\infty} V_{n}\left|P_{L}^{(n)}\right|^{2}, \quad V_{n}=\frac{4}{\pi^{2} n^{4}}\left[-1+\frac{N_{f}}{2}(n R m)^{2} K_{2}(n R m)\right] .
$$

Even at very large- $m$, as one dials $R$, it is possible to show that $V_{1}$ is positive for $R<R_{c}$ and center symmetry is stable. $V_{1}$ is negative for $R_{c}<R \lesssim \Lambda$. This calculable phase transition correspond to the lower lines in figures $2(\mathrm{~b})$ and $2(\mathrm{c})$. The lower phase boundary for $\mathrm{SU}(2) N_{f}$ flavor QCD(adj) for any $N_{f}<N_{f}^{a . f}$ is given by [38]

$$
m_{c} R_{c}^{\text {cont. }}=\{2.027,2.709,3.154,3.484\} \quad \text { for } \quad N_{f}=2,3,4,5
$$

The interesting aspect of the phase diagram is the non-decoupling of heavy fermions once the circle size is taken sufficiently small. In that regime, the fermion, despite being heavy, enters in the combination $m R$ and a sufficiently small $R$ makes the fermions behave as if they were light in that regime. The upper lines in these figures are incalculable by weak coupling methods, but it is essentially the deconfinement radius of pure Yang-Mills theory because massive fermions decouple in that regime. 


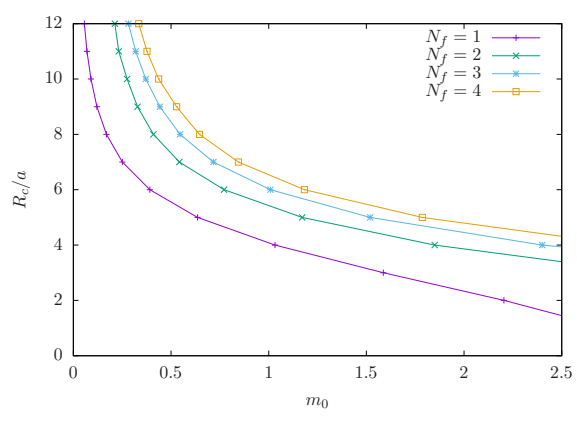

(a) Phase transition.

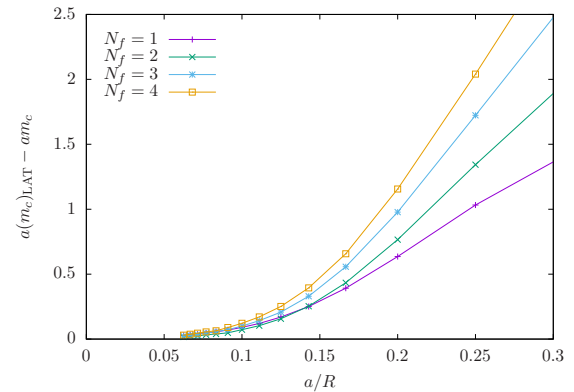

(b) Continuum limit.

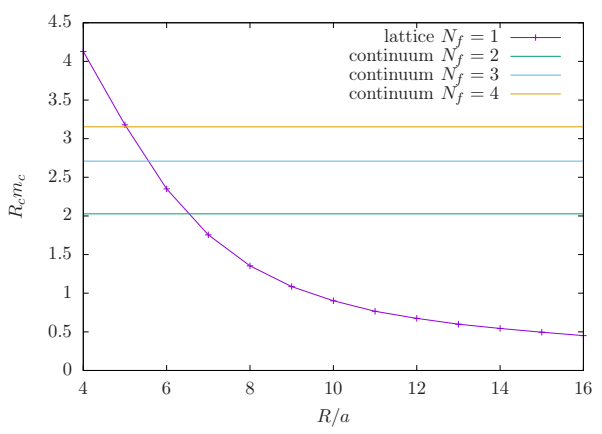

(c) Transition as function of $R / a$.

Figure 3. Figure (a): numerical determination of the critical radius $R_{c}$ for the deconfinement transition as a function of the mass in the one-loop approximation on the lattice from eq. (4.14) for $N_{f} \mathrm{QCD}$ (adj). In figure (b) the deviations of the critical mass $\left(m_{c}\right)_{\mathrm{LAT}}$ determined by the one-loop lattice effective potential from the expected continuum result $m_{c}$ (eq. (5.4)) are represented as a function of $a / R$. Keeping $R$ fixed, the difference tends to zero in the continuum limit. Figure (c) represents the transition for $N_{f}=1$ as a function of $R / a$ to compare $R_{c} m_{c}$ with the values of eq. (5.4).

Lattice: the expected deconfined regions on the lattice are shown for different $N_{f}$ in figure 3(a). Even for $N_{f}=1$, there is a perturbative transition line since lattice artefacts violate the exact cancellation between gluon and fermion loops, especially at scales close to the lattice cutoff $1 / a$. Such a significant deviation from the continuum phase diagram is expected for small $R / a$, while in the large $R / a$ regime a good agreement with the continuum predictions should be observed. The corresponding convergence to the continuum limit is shown in figure $3(\mathrm{~b})$. The deviations from the continuum phase diagram at $m_{0}>0$ and small $R / a$ are labeled as the "lattice pert. regime" in figure $2(\mathrm{~b})$. In this regime the theory behaves rather similarly to $\mathrm{QCD}(\mathrm{adj})$ for $N_{f}>1$ in the continuum with a confined phase that extends up to larger $m$ the smaller $R / a$ is. The appearance of this regime is in agreement with the result of our first numerical simulations [29].

The coefficient $V_{\text {eff }}^{(n)}$ in the perturbative effective Polyakov loop potential of SYM provides some further indications for the difference between lattice and continuum phases: the numerical solution shows that it monotonically decreases with $R / a$. In the continuum $V_{\text {eff }}^{(n)}=0$ at the perturbative level and due to the non-perturbative contributions it be- 
comes an increasing function of $R$. There is hence a competition between non-perturbative effects and perturbative lattice artefacts: the transition to the "lattice pert. regime" is indicated by a turnover from the increasing continuum to the decreasing lattice perturbative behaviour, a minimum of $V_{\mathrm{eff}}^{(n)}$ and a maximum of the susceptibility. The investigation of the continuity in SYM is limited to a certain range of $R / a$ before this turnover, where the lattice artefacts are under control. This is the limit of large $R / a$ while $R$ is kept small. Even outside this regime, the qualitative properties are not completely different. They can be compared to $N_{f}>1 \mathrm{QCD}(\mathrm{adj})$ in the continuum and the qualitative features should be similar.

The perturbative continuum regime is reached considering the limits $\beta \rightarrow \infty$ and $R / a \rightarrow \infty$, the continuum limit for an asymptotically free theory regularized on the lattice. In this limit there is a simple perturbative relation between the renormalized mass $m$ and the bare mass in lattice units $m_{0}$. A regime where the perturbative effective potential provides a reasonable approximation is also approached in the small $R / a$ limit at fixed lattice spacing. However, this is not strictly perturbative, in particular a non-perturbative renormalization of the mass is required. The mass parameter $m_{0}$ in the perturbative formulas is replaced by $m / a$ fixed by some renormalization condition, in our case a fixed value of the adjoint pion mass in the large $R$ limit. The fixed scale approach that we apply in our current study allows to keep the line of constant physics, i. e. constant renormalized parameters, unambiguously, compared to our previous studies in [29].

\subsection{Explanation of the discrepancy of lattice and continuum}

The phase diagram of lattice SYM with Wilson fermions and Wilson parameter set to $r=1$ exhibits a different phase structure than in the continuum limit. In particular, for sufficiently small-bare mass, the lattice theory is always confined and for a large mass, it is confining at large $R$, deconfined in an intermediate regime, and confined again at small- $R$, as shown in figure 2(b). Note that the lattice regularization neither respects supersymmetry, nor chiral symmetry. In fact, if it were to respect one, it would respect the other as the only relevant chiral symmetry breaking operator $m \bar{\lambda} \lambda$ is also the only supersymmetry breaking relevant operator for this system.

Here, we explain this discrepancy in more detail, and argue that both of these conclusions are actually correct, and that there is no contradiction between the two results.

Recall that both the continuum and lattice study aim to understand phase structure of the theory by using

$$
\tilde{Z}(R, m)=\operatorname{tr}\left[e^{-R \hat{H}(m)}(-1)^{F}\right]
$$

In continuum, for $m=0, \tilde{Z}(R, 0)$ is the supersymmetric Witten index, independent of $R$. Hence, there is no phase transition on the $m=0$ axis for any value of $R$. In the small- $R$ regime, there is no perturbatively induced potential for Wilson line.

There are non-perturbative monopole-instantons with two fermion zero modes $\mathcal{M}_{a}=$ $e^{-S_{m}} e^{-\vec{\alpha}_{a} \cdot(\vec{\phi}-i \vec{\sigma})}\left(\vec{\alpha}_{a} \lambda\right)^{2}$ where $\vec{\phi}$ is the holonomy field and $\vec{\sigma}$ is the dual photon. The crucial point is that despite the fact that monopole-instanton couple to holonomy field, it does not induce a boson potential as it has fermion zero modes. Rather, it does induce a chiral 
condensate as well as a superpotential. Note that $\prod_{a=1}^{N_{c}} \mathcal{M}_{a}=I_{B P S T}=e^{-S_{I}}(\operatorname{tr} \lambda \lambda)^{N_{c}}$, the usual $4 \mathrm{~d}$ instanton. Therefore, $\mathcal{M}_{a}$ can be considered as fractional instanton. The second order effects in the semi-classical expansion are magnetic bions $\left[\mathcal{M}_{a} \overline{\mathcal{M}}_{a+1}\right]$ and neutral bions $\left[\mathcal{M}_{a} \overline{\mathcal{M}}_{a}\right]$. In combination, these two types of bions induce mass gap for boson fluctuations (which are otherwise gapless to all orders in perturbation theory), and stability of center symmetry.

The bosonic potential for $\mathrm{SU}(2) \mathrm{SYM}$ is:

$$
\begin{aligned}
V^{\text {pert. }}(\phi) & =0 \\
V^{\text {non.pert. }}(\phi) & =e^{-2 S_{m}} \cosh \left(\frac{4 \pi}{g^{2}}(2 \phi-\pi)\right) \quad S_{m}=\frac{S_{\text {instanton }}}{N_{c}}=\frac{8 \pi^{2}}{g^{2} N},
\end{aligned}
$$

which has a minimum at $\phi=\frac{\pi}{2}$. Once a small mass for the fermion is added to the SYM action, (5.5) is still a well-defined object, however it is no longer the Witten index as the supersymmetry is explicitly broken. It can be interpreted as a twisted/graded partition function in which boson and fermion states are added according to a $\mathbb{Z}_{2}=(-1)^{F}$ grading.

Once the fermion mass is different from zero, there is a perturbatively induced GPY potential, and monopole-instanton effects also induce a potential for the Wilson line holonomy as the soft mass lifts the fermion zero modes. Both of these effects are center-destabilizing and proportional to the soft-SUSY breaking parameter $m$, while the neutral bion effects are center-stabilizing. The competition between these three effects, neutral bions vs. perturbative and monopole-instanton effects is responsible for the existence of the center and chiral phase transition. Also note that as $R$ gets smaller, the center breaking effects certainly dominate, with no chance for the center-symmetry to stabilize again.

On the other hand, lattice SYM is quite different. The phase diagram shown on figure 2(b) is much like the continuum analysis of the $1<N_{f}<N_{f}^{*}$ theories. The reason for such a difference is the following. On the lattice, the dispersion relation of free bosons and fermions are not equal. The inverse propagator reads:

$$
\begin{array}{cl}
\text { bosons : } & \hat{p}^{2}=\sum_{i=1}^{4} 4 \sin ^{2}\left(\frac{p_{i}}{2}\right) \\
\text { Wilson fermion : } & \hat{\hat{p}}^{2}+\left(m_{0}+\frac{r}{2} \hat{p}^{2}\right)^{2}, \quad \hat{\hat{p}}^{2}=\sum_{i=1}^{4} \sin ^{2} p_{i} .
\end{array}
$$

If the Wilson term is set to $r=1$, indeed, there are no doublers but rather interestingly, a lattice Wilson fermion, in its effect to center-symmetry realization and to the potential (4.14) is more powerful than a single continuum fermion! The lattice theory with $N_{f}=1$ behaves similarly to $1<N_{f}<N_{f}^{*}$ continuum theories.

In this sense, $N_{f}^{\text {lattice }}=1$ is capable to undo the center-breaking effect of gauge fluctuations even at one-loop order. The Wilson term is the reason why center-symmetry is stable at one-loop order in lattice perturbation theory in the $m_{0}=0$ limit,

$$
V^{\text {pert. }}(\phi)=N^{2} \sum_{n=1}^{\infty} V_{n}\left|P_{L}^{(n)}\right|^{2}, \quad V_{n}>0 .
$$


The lattice center-stabilizing effects given by Wilson fermions continue to hold true for sufficiently small bare fermion mass $m_{0}$. Furthermore, for very large- $m_{0}$, as one dials $R$, it is possible to show that $V_{1}$ is positive for $R<R_{c}$ and center symmetry is stable and $V_{1}$ is negative for $R_{c}<R \lesssim \Lambda$. Such a calculable phase transition corresponds to the lower line in 2(b). The upper line is incalculable by weak coupling methods, but it is essentially the (inverse) deconfinement temperature of pure Yang-Mills theory. Note that fermions play a two fold role here. The interesting aspect is the non-decoupling of heavy fermions once the radius is taken small. In that regime, the fermion, despite being heavy, enters in the combination $m_{0} R$ and sufficiently small $R$ makes the fermions behave as if they were light. When $R$ is sufficiently large, heavy fermions essentially decouple from the dynamics and we have the transition of pure Yang-Mills theory.

The observation of the non-decoupling of heavy fermions can be phrased in a different way: at each $N_{t}=R / a$ there is a fixed value of $m_{0}=a m$ for the transition. As a function of $a$ in simulations at fixed $N_{t}$, the transition line follows a constant $m_{c} R_{c}$ like $N_{f}>1$ QCD(adj) in the continuum. This fixed $N_{t}$ approach has been applied in our previous simulations and the results show indeed a narrowing of the deconfined region towards smaller $a$, i. e. larger $\beta$ [29]. The continuum limit hence requires the large $N_{t}$ limit. At fixed $N_{t}=4$, lattice $N_{f}=1 \mathrm{QCD}(\mathrm{adj})$ is comparable to $N_{f}>4$ in the continuum, see figure 3(c). Note that this simple relation holds only at the one loop level.

The fermion-boson mismatch of the dispersion relations is essentially the reason why the lattice theory with $N_{f}^{\text {lattice }}=1$ behaves differently from the continuum theory with $N_{f}=1$. In the continuum limit, tuning towards the supersymmetric point, the lower transition line in figure 2(b) tends towards $R=0$ and the continuum phase diagram shown in figure $2(\mathrm{a})$ is recovered.

In the next section, we present evidences from Monte-Carlo simulations giving rise to the phase diagram figure 2(b), providing a numerical proof of the idea of adiabatic continuity between the small and large radius for sufficiently light fermions. Furthermore, we will also provide evidence for the continuity of the phase transition lines between the weak coupling calculable regime and strong coupling incalculable regime.

\section{$6 \quad$ Numerical results for compactified adjoint QCD}

We have performed numerical simulations of $\mathrm{SU}(2) \mathrm{QCD}(\mathrm{adj})$ with thermal and periodic boundary conditions for different fermion masses and couplings. Most of our simulation are for $N_{f}=1$ but we have also considered $N_{f}=2 \mathrm{QCD}$ (adj) in an exploratory study. The main focus are the Polyakov loop, its susceptibility, and the Binder cumulant, to investigate the theoretical conjectures about the deconfinement transition. In the fixed scale approach the compactification radius $R$ is proportional to the number of lattice sites in the temporal direction $N_{t}=R / a$. Since the lattice spacing is fixed, we can disentangle easily the renormalization effects and the tuning of the bare fermion mass from the change of the compactification radius. Based on our previous experience, a rather small lattice spacing is required since the lattice itself is a relevant source of supersymmetry breaking, even more relevant than the breaking induced by the gluino mass. For this reason we have compared different lattice actions. 


\subsection{Confined and deconfined phases in SYM theory}

The Polyakov loop and its susceptibility provide a signal for a possible deconfinement transition. Typically the transition can be identified by the peak of the susceptibility and by its divergence in the thermodynamic limit. Here a special care is required since the expected regime at small compactification radii is characterized by large fluctuations of the Polyakov loop, which are unrelated to the fluctuations between the confined and deconfined phases. The expectation value of $\left|P_{L}\right|$ and its susceptibility can become large without a deconfinement phase transition for periodic boundary conditions just because of the almost flat effective potential. A precise estimation of the phase of the theory can be obtained from the histogram of the Polyakov loop and from the Binder cumulant.

The Polyakov loop and its susceptibility are shown in figure $4 .{ }^{5}$ As expected, thermal boundary conditions lead to a deconfinement transition at $R / a \sim 7$ indicated by a steep rise of the Polyakov loop expectation value and by a peak of the susceptibility. The Polyakov loop expectation value for periodic boundary conditions remains rather small, but a large value of the susceptibility is observed at $R / a \sim 5$. However, even close to the maximum, the histogram of $P_{L}$ is still peaked at zero as in the confined phase. The large values of the susceptibility originate from a broadening of the Polyakov loop distribution but not from a phase transition, see figure 5 . The constraint effective potential becomes nearly flat, but its minimum remains at zero. At the smallest $R / a$ the susceptibility decreases again, a behaviour compatible with the predictions of lattice perturbation theory. The Binder cumulant shows further evidences for such a scenario. If thermal boundary conditions are chosen for the gluino, $B_{4}$ crosses the critical value of the $Z_{2}$ Ising universality class. Instead, the Binder cumulant never reaches this value in the periodic case, see figure $4(\mathrm{c})$, and it decreases toward zero at the smallest $R / a$, in agreement with the perturbative predictions.

We have simulated the theory also with a different lattice action and a different lattice spacing and obtained compatible results, see figure 6 . In this case a larger volume would be required for a clearer identification of the thermal deconfinement transition from the peak of the susceptibility. The transition at $T_{c}$ corresponding to $R / a \sim 12$ is more clearly identified by the Binder cumulant 6(c). The phase diagram is in good agreement with figure 4: for periodic boundary conditions very large peak value of the susceptibility is obtained at rather small compactification radius $R / a=R_{p} / a \sim 6$ and the susceptibility decreases at smaller $R$. The separation of the peak of the susceptibility for periodic boundary conditions from the one of the thermal transition becomes significantly larger at the smaller lattice spacing. This indicates that $R_{p} T_{c}$ becomes smaller and might even, as expected for continuity, vanish in the continuum limit. The absence of a confined phase in periodic compactified SYM is quite stable with respect to a change of the gluino mass.

\footnotetext{
${ }^{5}$ In lattice simulations, it is a common practice to look at $\left\langle\left|P_{L}\right|\right\rangle$ instead of $\left\langle P_{L}\right\rangle$. The reason for the absolute value is the fact in finite volume and finite $N_{c}$, there are no strict phase transitions. The transitions between different vacua which is forbidden for the theory on $\mathbb{R}^{3} \times S^{1}$, upon compactification down to $T^{3} \times S^{1}$, is replaced by $e^{-\operatorname{Vol}\left(T^{3}\right)}$. This indeed leads to spontaneous symmetry breaking as $\operatorname{Vol}\left(T^{3}\right) \rightarrow \infty$. If the simulation time is much larger than the inverse tunneling probability, the Polyakov loop expectation value will average out to zero even in the deconfined phase. This will not take place if one uses $\left\langle\left|P_{L}\right|\right\rangle$, giving a cleaner diagnostic of confinement-deconfinement transition in finite volume.
} 


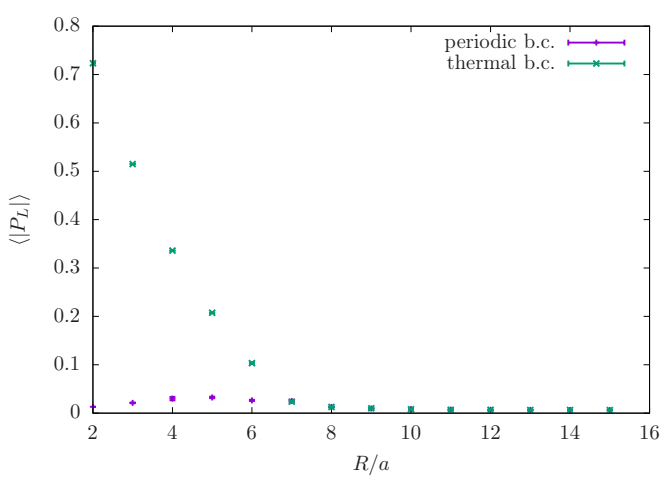

(a)

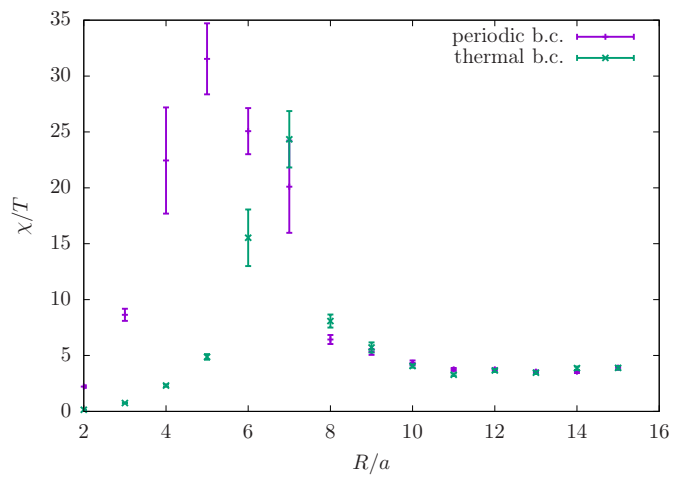

(b)

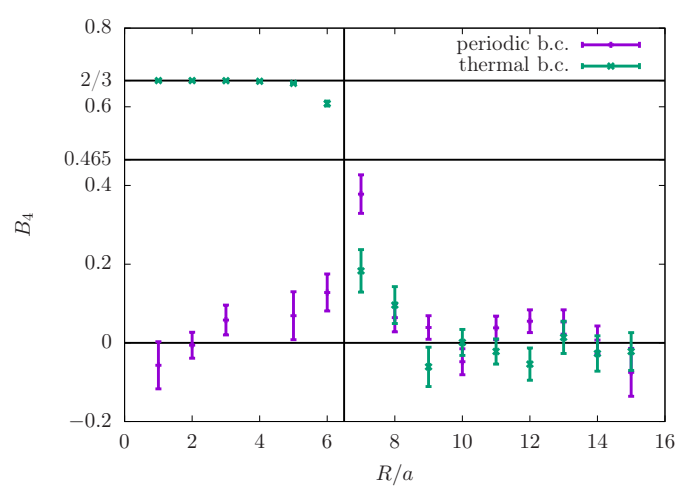

(c)

Figure 4. The results for the Polyakov loop at $\beta=1.65, \kappa=0.1750, c_{s w}=1.0$ on a $16^{3} \times N_{t}$ lattice as a function of $N_{t}=R / a$. The plots show a comparison of periodic and thermal fermion boundary conditions: (a) the average modulus of the Polyakov loop $\left|P_{L}\right|$, (b) the susceptibility of $P_{L}$, and (c) the Binder cumulant. The "pion mass" (gluino-ball) at these parameters in lattice units is $a m_{\pi}=0.64631(67)$. The vertical line in (c) indicates the point of the deconfinement transition.

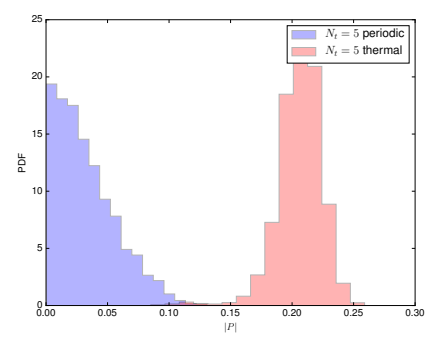

(a)

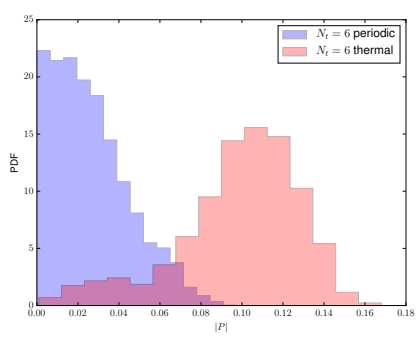

(b)

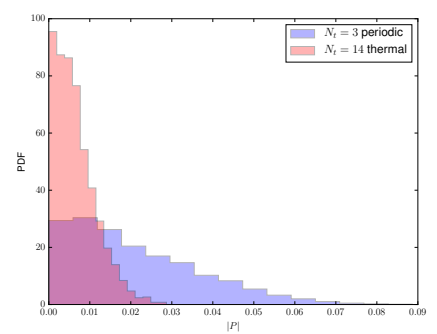

(c)

Figure 5. The histograms for the Polyakov loop at selected $N_{t}=R / a$ for periodic and thermal fermion boundary conditions. The parameters are the same as in figure 4. 


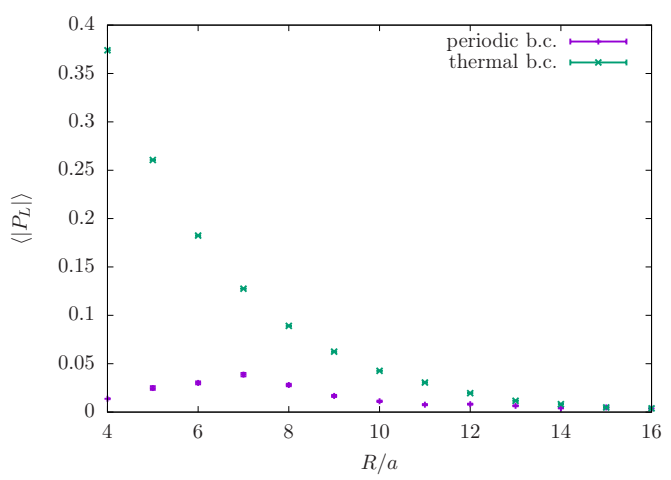

(a)

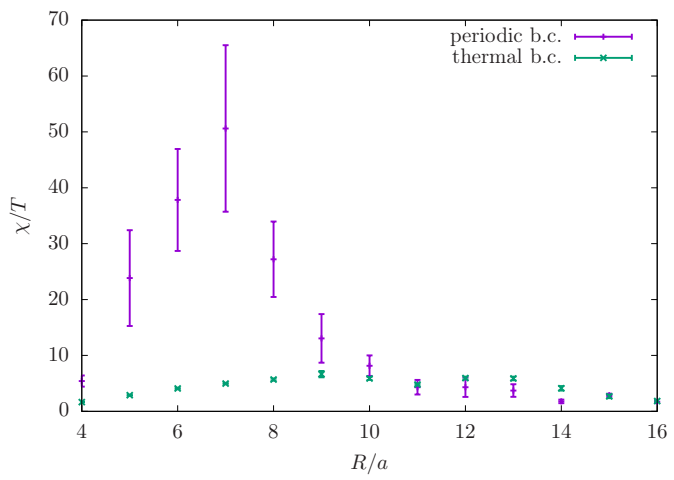

(b)

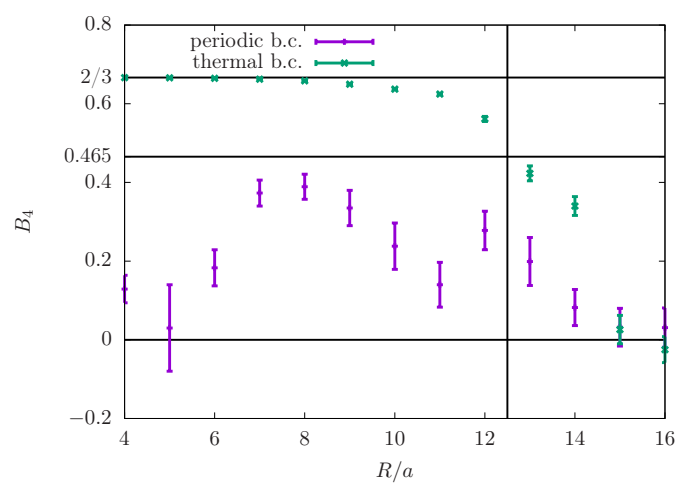

(c)

Figure 6. The results for the Polyakov loop at $\beta=1.75, \kappa=0.14925, c_{s w}=0$ on a $24^{3} \times N_{t}$ lattice as a function of $N_{t}=R / a$. The plots show a comparison of periodic and thermal fermion boundary conditions in the same way as in figure 4: (a) the average modulus of the Polyakov loop $\left|P_{L}\right|$, (b) the susceptibility of $P_{L}$, and (c) the Binder cumulant. In these simulations one level of stout smearing was applied to the gauge links in the Dirac operator. The physical observables at these parameters can be found in [31].

\section{2 $N_{f}=2 \mathrm{QCD}(\mathbf{a d j})$}

There is a limited knowledge about the properties of $N_{f}=2 \mathrm{QCD}(\operatorname{adj})$ at zero temperature. Recent numerical investigations have shown some evidence that the model could be already near the conformal window [49], see also [24] for a recent theoretical analysis. The running of the strong coupling has been found to be, however, quite different from zero [50]. The spectrum of the theory has a light $0^{++}$glueball and an heavier pion(gluino-ball), while the opposite occurs in the QCD bound spectrum. This theory is free from the Pfaffian sign problem, but a near conformal behavior requires rather large volumes to be safe from finite volume corrections.

In the numerical simulations of this theory we have found a clear signal of a deconfined intermediate phase and confined phases at large and small $R / a$, see figure 7 . The deconfinement can be clearly identified by the histogram in figure $7(\mathrm{c})$. The different theories 


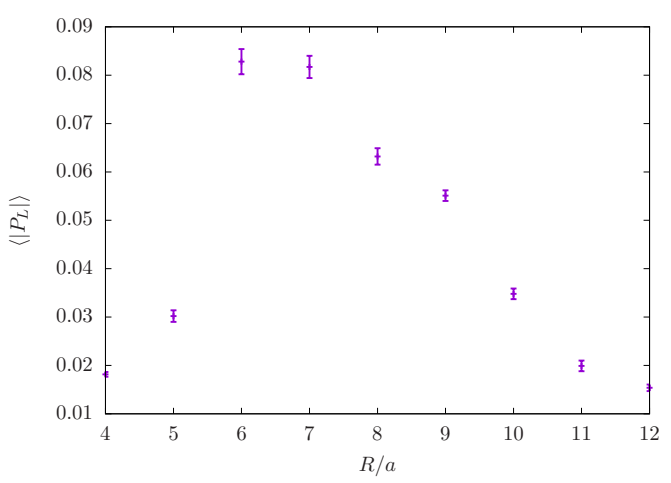

(a)

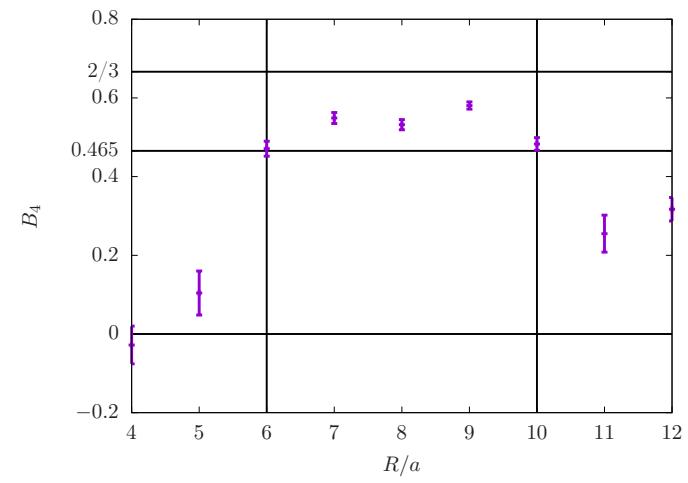

(b)

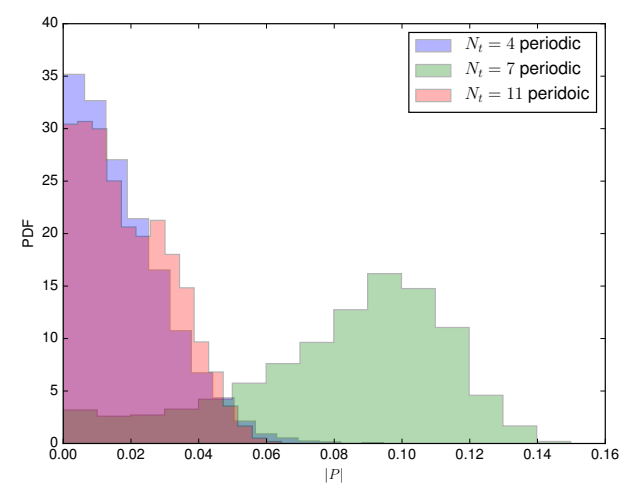

(c)

Figure 7. Polyakov loop data from simulations of $N_{f}=2 \mathrm{QCD}(\operatorname{adj})$ at $\beta=1.75, \kappa=0.1620$ $\left(c_{s w}=1.0\right)$ with periodic boundary conditions. (a) The average modulus of the Polyakov loop as a function of $R / a,(\mathrm{~b})$ the Binder cumulant, (c) the histogram of $\left|P_{L}\right|$.

can not be directly compared with each other, but in the simulations we have considered so far we never observed the disappearance of the intermediate phase. The intermediate phase is expected in general for heavy fermion masses, but for a conformal theory it should persist even for arbitrary low fermion masses, compare figure 2(c). It is hard to judge about the conformal behavior based on our present simulations, but at least they are not contradicting it.

The extension of the intermediate deconfined phase seems to be related to different competing effects. The one-loop calculation for Polyakov loop suggests that the confined phase at small $R$ for a given $m$ becomes larger the larger $N_{f}$ is, which implies a shrinking deconfined phase with $N_{f}$. Fermions enforce center-stability and confinement in the perturbative small $R$ regime. On the other hand, fermions induce screening effects in the $\beta$ function. In the chiral limit, the leading order coefficient of the beta function is $\beta_{0}=\left(\frac{11}{3}-\frac{2}{3} N_{f}\right) N$. Therefore, fermion fields also counteract confinement at large $R$ due to screening effects. Our results tend to indicate that the sum of these effects prefers the extension of the deconfined phase, but further analysis and a more detailed consideration 
of finite volume effects are required in order to make a clearer statement about the continuity at $N_{f}=2$. Further studies of the two-flavor theory might be interesting in particular regarding the conjectures of [24] about the chiral transition.

\subsection{Eigenvalue distributions and Abelian vs. non-abelian confinement}

In this section we consider the distribution of the Polyakov line eigenvalues in SYM with the same parameters as in figure 6. As discussed in section 4.2, the per-site distribution corresponds to the per-site constraint effective potential. It is different from the effective action of the volume averaged Polyakov eigenvalue phase and in the strong coupling limit it becomes equivalent to the Haar measure. We consider the probability function $P_{\mathrm{pdf}, \phi}(\phi)$ of finding a Polyakov line at an arbitrary spacial point $\mathbf{x}$ whose eigenvalues are $\exp ( \pm i \phi(\mathbf{x}))$ in a random configuration of the Markov chain.

Abelian vs. non-Abelian confinement: the constraint effective potential obtained from the distributions is shown in figure 8. In case of thermal boundary conditions it is similar to the pure gauge case [51]. The asymmetric form above the deconfinement transition indicates a positive or negative expectation value of the Polyakov line. In the periodic case the plain probability function does not show a considerable dependence on the compactification radius $R / a$. The deviations become much clearer if the per-site distribution is normalized by the Haar measure, see figure 9 . Due to numerical instabilities, the division by the Haar measure produces some artificial distortion in the regions where it is small. There are two possible mechanism which may render $\left\langle P_{L}\right\rangle=0$ in SYM compactified on a circle and both of these are taking place in the theory, albeit at different regimes, that we refer to as non-Abelian and Abelian confinement.

- Random distribution: the flat distribution observed at large compactification radii is compatible with the non-Abelian confinement picture: random fluctuations of the phase $\phi$ in a nearly flat effective potential are the origin of the a vanishing expectation value for the Polyakov loop.

- Abelianizing distribution: at small $R / a$ the behaviour might be identified with the picture of Abelian confinement, where confinement is produced by a distribution of $\phi$ centered around the zeros of $\cos (\phi)$.

It is remarkable that the theory up to a very small radius $R \sim 2 / T_{c}$ shows a distribution compatible with the strong coupling limit. ${ }^{6} \mathrm{SYM}$, and more general QCD(adj), as well as center-stabilized Yang-Mills and QCD theories are expected to possess both regimes, an Abelian confinement regime where adjoint Higgsing is operative, and a strong coupling regime where random fluctuation of the phase of the Polyakov loop take over. Our numerical results provide evidence for the continuity between these two regimes.

Any constraint effective potential obtained from the distribution of the order parameter is symmetric under center symmetry on a finite volume in the limit of infinite statistic.

\footnotetext{
${ }^{6}$ We thank Misha Shifman for discussions on random vs. abelianizing distributions and on the connections of non-Abelian vs. Abelian confinement.
} 


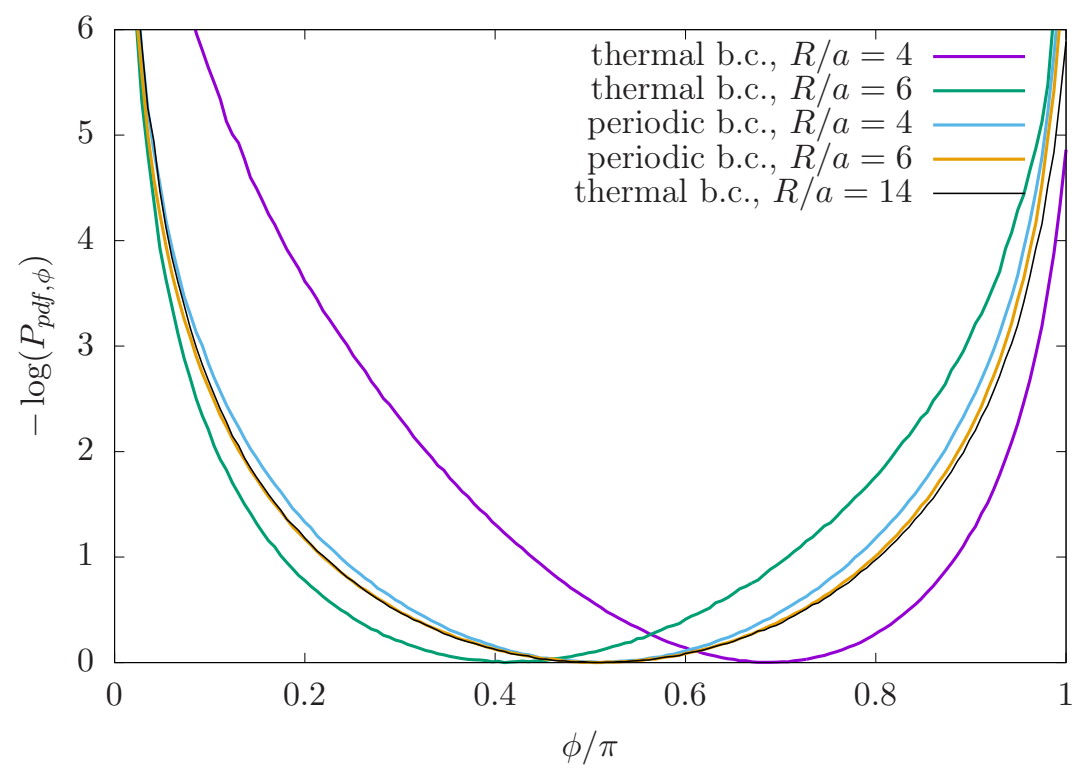

Figure 8. Constraint effective potential from the per-site distribution of the Polyakov phase $\phi$ comparing periodic and thermal fermion boundary conditions. The minimum at $\phi>\pi / 2$ and $\phi<\pi / 2$ for $N_{t}=4$ and $N_{t}=6$ in the thermal case indicates the negative and positive average value of the Polyakov line in the center broken phase. The histogram has been determined the same runs as in figure 6 . The plot in this representation can be directly compared to the pure gauge case [51].

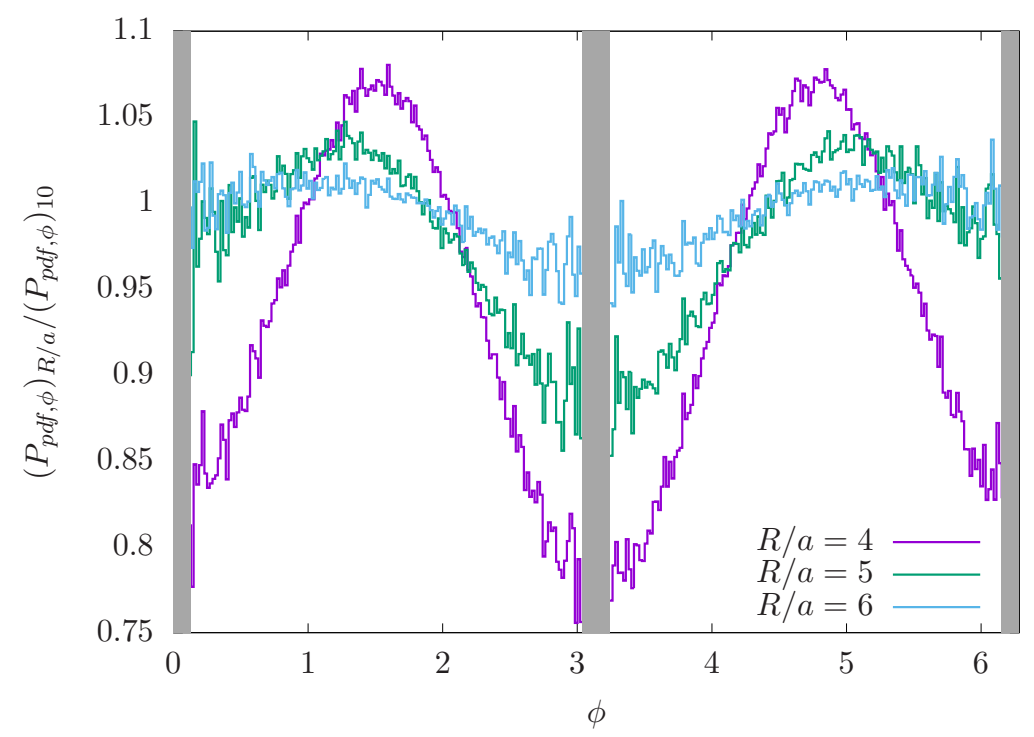

Figure 9. Per-site distribution of the Polyakov phase $\phi$ for different $R / a$ normalized with respect to the distribution at $R / a=10$, which is close to the Haar measure. The histogram has been determined the same runs as in figure 6 . The normalization of the histogram generates large errors in the regions close to $0 \bmod \pi$, corresponding to the gray bands in the plot. These regions have been excluded. 


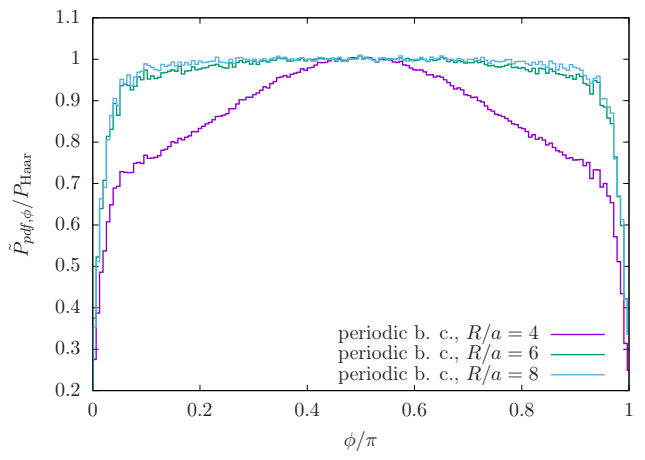

(a)

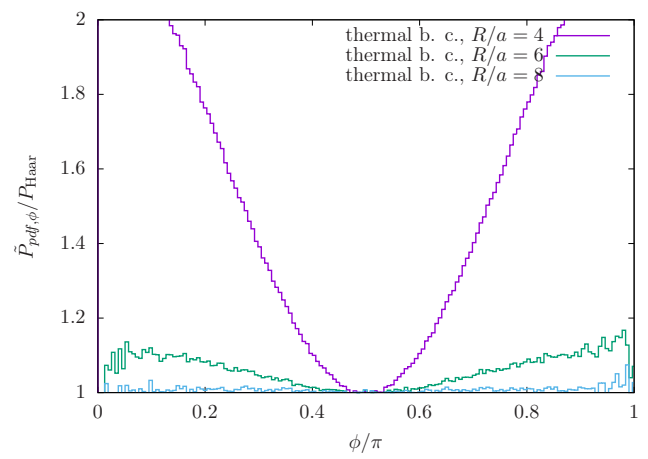

(b)

Figure 10. Symmetrized per-site distribution of the Polyakov phase $\phi$ normalized with respect to the Haar measure for different $R / a$. The histogram has been determined the same runs as in figure 6 for periodic (figure (a)) and thermal (figure (b)) fermion boundary conditions.

The spontaneous breaking of center symmetry appears in the constraint effective potential of the volume averaged order parameter in terms of two minima related by the broken symmetry. The barrier between these minima diverges in the infinite volume limit and this signal is stable under a symmetrization of the distribution. Such an effect does not appear in the per-site distributions. With a finite statistics and spontaneously broken center symmetry the non-zero average Polyakov loop will lead to asymmetric distributions as in figure 8 , but a symmetrized distribution will in general not show a double peak. Nevertheless it is instructive to investigate the symmetrized per-site distributions in order to qualify the difference to the Haar measure. Symmetrization of the distributions with respect to center symmetry means $\tilde{P}_{\mathrm{pdf}, \phi}(x)=\frac{1}{2}\left(P_{\mathrm{pdf}, \phi}(x+\pi)+P_{\mathrm{pdf}, \phi}(x)\right)$ for $\mathrm{SU}(2)$. The symmetrized distributions normalized to the Haar measure are shown in figure 10. The difference between the preference of the confined or deconfined minima compared to the Haar measure can be clearly observed.

Information about the distribution of the volume averaged $\phi$, which is more directly related to the effective potential, can be obtained from the susceptibility of this observable. Like for the case of the traced Polyakov line in figure 6 , there is a peak at a certain $R / a$ that indicates the flattening of the effective potential, but not a phase transition. It is expected that the maximum of the susceptibility moves to smaller and smaller $R / a$ in the continuum limit and the effective potential becomes flat (up to non-perturbative contributions) at small $m$ and $R$.

In contrast to the expected continuum behaviour, we observe at very small $R / a$ decreasing susceptibilities of $P_{L}$ and $\phi$ at the same time, together with a narrowing of the per-site distribution divided by the Haar measure. Such regime corresponds to the "lattice pert. phase" in figure 2(b) where the fluctuation of $\phi$ are suppressed. It is a lattice artefact, but it does not break the continuity picture since the theory becomes just similar to QCD(adj) at larger $N_{f}$. The region where compactified lattice SYM is most similar to continuum SYM at small $R$, for what concerns the vanishing perturbative contributions, is close to the maximum of the susceptibility. 


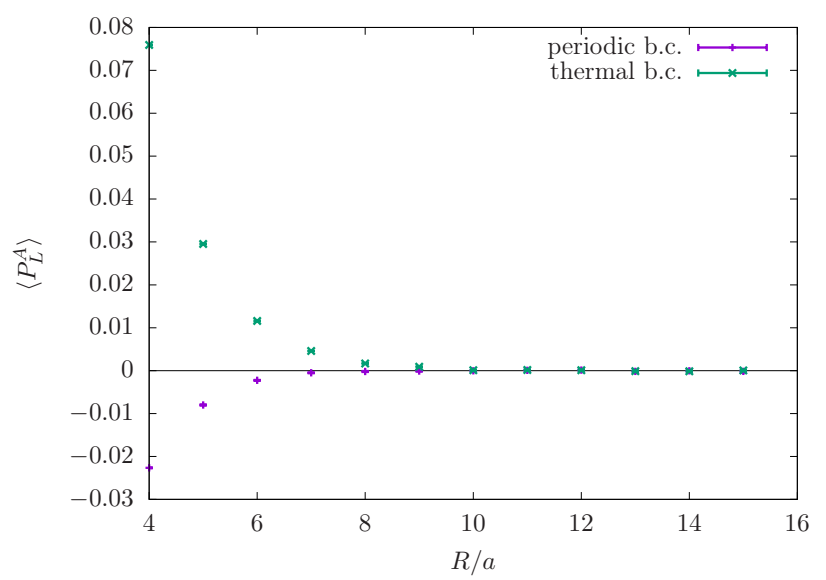

Figure 11. Polyakov loop in the adjoint representation as a function of the compactification radius for periodic boundary conditions with the same parameters as in figure 6 .

\subsection{The Polyakov loop in the adjoint representation}

In addition to $P_{L}$, we have also measured the expectation value of the Polyakov loop in the adjoint representation. Even at zero temperature, it is expected to have a non-vanishing expectation value since it is not fixed by center symmetry. It is interesting to note that for periodic boundary conditions the adjoint Polyakov loop develops a negative expectation value at small $R / a$, while the Polyakov loop in the fundamental representation is always close to zero, see figure 11. The adjoint Polyakov loop for thermal boundary condition develops, on the other had a positive expectation value.

The non-vanishing expectation value of the adjoint Polyakov loop can be approximately related to perturbative effective potential and the behavior of the per-site distribution of $\phi$. On each site the phase $\phi(\mathbf{x})$ determines the trace of the fundamental Polyakov line as $\cos (\phi)$ and the adjoint one as $1+2 \cos (2 \phi)$. One can assume that the expectation values are basically determined by the per-site distribution of $\phi$ :

$$
\begin{aligned}
\left\langle P_{L}\right\rangle & \approx \frac{1}{2} \int_{0}^{\pi} 2 \cos (\phi) P_{\mathrm{pdf}, \phi}(\phi) d \phi, \\
\left\langle P_{L}^{A}\right\rangle & \approx \int_{0}^{\pi}(1+2 \cos (2 \phi)) P_{\mathrm{pdf}, \phi}(\phi) d \phi .
\end{aligned}
$$

The probability distribution function $P_{\mathrm{pdf}, \phi}(\phi)$ at strong coupling (larger $R / a$ ) is proportional to the Haar measure. In this case both integrals are zero, meaning a vanishing expectation value of both in the adjoint and in the fundamental representation. At smaller $R / a$, the probability distribution found in section 6.3 can be well described by a functional of the form

$$
K-\cos (2 \phi)+\alpha \cos (4 \phi),
$$

where $\alpha$ is a small positive constant, $0 \leq \alpha<0.5$. The term proportional to $\cos (4 \phi)$ still vanishes in the integral (6.1), but the integral (6.2) is now equal to

$$
\left\langle P_{A}\right\rangle=\frac{\alpha}{\alpha-1}<0,
$$


implying again a negative value of the adjoint Polyakov loop. However, this is only a crude approximation that becomes exact only in the strong coupling limit. The main effect is due to a localization (Abelianization) of the distributions: the random distribution of $\phi$ according to the Haar measure leads to $\left\langle P_{A}\right\rangle=0,\left\langle P_{L}\right\rangle=0$. In the completely localized limit, on the other hand, $\phi$ can be set to the perturbative minimum $\phi=\frac{\pi}{2}$ for periodic and $\phi=0$ for thermal boundary conditions. In this saddle point approximation, the values are $\left\langle P_{A}\right\rangle \neq 0,\left\langle P_{L}\right\rangle=0$ for the periodic and $\left\langle P_{A}\right\rangle \neq 0,\left\langle P_{L}\right\rangle \neq 0$ for the thermal case. SYM in the continuum has only a non-perturbative localization of the distributions. Lattice artefacts provide localization effects even at the perturbative level, but still the numerical results show only a small non-zero value of $\left\langle P_{A}\right\rangle$ at the smallest $R / a$.

\subsection{The chiral condensate}

The chiral condensate provides information about the spontaneous chiral symmetry breaking as a function of $R / a$. The bare condensate corresponds to the derivative of the partition function with respect to $m$,

$$
\langle\bar{\lambda} \lambda\rangle_{B} \doteq-\frac{1}{R V_{3}} \frac{\partial}{\partial m} \log (Z(\beta, m)) .
$$

A rough one-loop perturbative prediction can be computed from a derivative of the effective potential with respect to $m$ setting $\phi$ to the value of the minimum, corresponding basically to the trace of the fermion propagator in the given gauge-field background. In this perturbative limit one obtains rather small values that are decreasing with $R / a$ and proportional to the mass term including the Wilson mass.

Recall that since the Wilson fermion action does not respect chiral symmetry, the condensate receives both additive and multiplicative renormalization. The subtracted condensate,

$$
\langle\bar{\lambda} \lambda\rangle_{S}=\langle\bar{\lambda} \lambda\rangle_{B}^{R}-\langle\bar{\lambda} \lambda\rangle_{B}^{R=R_{r}}
$$

is considered to remove the additive renormalization. The subtraction is performed with respect to a fixed radius $R_{r}$, in the present case $R_{r} / a=4$ and thermal boundary conditions. The transition from the low temperature broken phase with a non-vanishing expectation value of the condensate to the unbroken phase is indicated by a jump of $\langle\bar{\lambda} \lambda\rangle_{S}$. The subtracted condensate as a function of $N_{t}=R / a$ is shown in figure 12 for periodic and thermal boundary conditions. In the thermal case there is a signal for a chiral transition around the same point as the deconfinement transition. It can be identified by the peak of the chiral susceptibility. The considerable decrease of the thermal $\langle\bar{\lambda} \lambda\rangle_{S}$ at very small $N_{t}$ is an indication of lattice artefacts introduced by the Wilson fermion discretization. The chiral susceptibility remains small in this region, which indicates the absence of a physical phase transition.

The subtracted chiral condensate for periodic boundary conditions is close to its large$R$ value up to a small radius $R / a \sim 6$. At a smaller radius there is a decrease of the subtracted chiral condensate, in agreement with the perturbative prediction, where lattice artefacts introduce a significant distortion even in the case of thermal boundary conditions. The decreasing expectation value of the chiral condensate might also be in relation to 


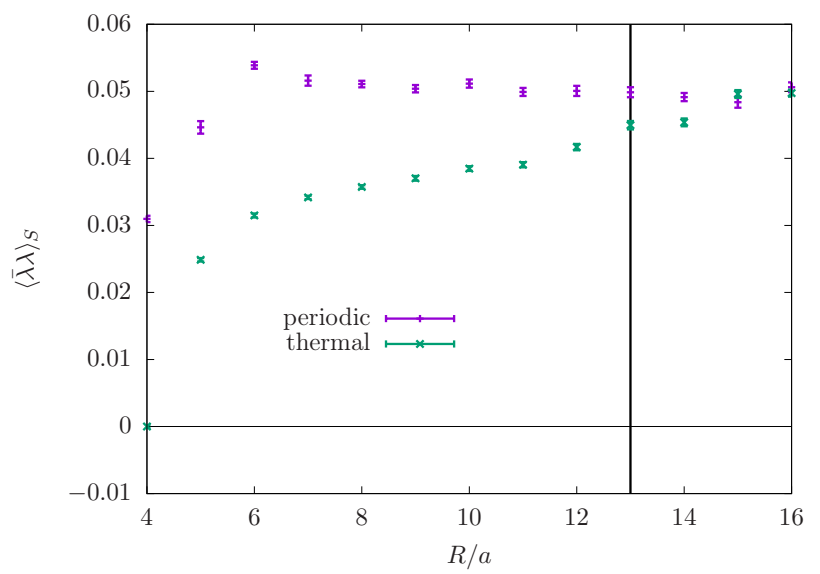

Figure 12. Subtracted chiral condensate as a function of the compactification radius for periodic and anti-periodic boundary conditions with the same parameters as in figure 6 . The black vertical line indicates the maximum of the chiral susceptibility for anti-periodic boundary conditions. Note the persistence of a non-vanishing chiral condensate when fermions are endowed with periodic boundary condition up to very small circle size.

the adjoint Polyakov loop as found in [52]. Our findings can also be compared to the investigation of the chiral order parameter with adjoint staggered fermions at larger $N_{f}$ and periodic boundary conditions in [53], where it was found that the chiral symmetry remains spontaneously broken to small $R$ with an indication for a chiral symmetry restoration at very small $R$.

The crucial point of figure 12 is that unlike thermal compactification, a non-vanishing chiral condensate persists up to rather small circle size in the circle compactication. In fact, as long as center-symmetry is intact, chiral symmetry breaking remains robust. This provides further evidence for the idea of adiabatic continuity.

\section{Conclusions}

Our study of compactified $\mathrm{QCD}(\operatorname{adj})$ on the lattice has extended the present knowledge of the phase diagram of this class of theories and our previous analysis in several aspects. We have focused in particular on $\mathcal{N}=1$ supersymmetric Yang-Mills theory. We have analyzed the lattice theory in the perturbative setup and determined in particular the deviations from continuum perturbation theory. Already in the perturbative calculations, there is a clear difference between the lattice and the continuum predictions for the phase diagram. From these calculations we have conjectured the phase diagram of QCD (adj) on the lattice and found good agreement with the observations in our first results [29]. These first investigations have been limited by the difficult issue of renormalization of the bare parameters. Therefore we have chosen a fixed scale setup in the present investigations.

We have measured a number of different observables in order to characterize the different phases that appear as a function of the compactification radius $R$. Up to small $R$ our observations are in agreement with the analytical predictions: there is no phase transition 
and a flattening of the effective potential of the Polyakov line is observed towards smaller $R$. Chiral symmetry remains spontaneously broken in the confined phase up to small $R$.

At very small $R$ there is a phase with considerable deviations from the continuum predictions. The fermion discretization leads to a behaviour expected for a larger number of fermion flavours in the continuum. Decreasing $R$, a continuous extension of the deconfinement transition line towards smaller $m$ is expected for continuum supersymmetric Yang-Mills theory. On the lattice the confined phase extends instead to the large $m$ limit when $R$ tends to zero at a fixed lattice spacing. A peak in the Polyakov line susceptibility that is not related to deconfinement indicates the transition to this phase with considerable discretization effects. We have found indications that the deviation between the thermal deconfinement transition and this peak of the susceptibility increases towards the continuum limit. If this observation can be further substantiated, it would confirm the continuity of the deconfinement transition and the validity of the semiclassical analysis. The appearance of the lattice artefact phase, characterized by a decreasing chiral condensate and a negative adjoint Polyakov loop, can be understood already from a perturbative analysis.

Despite the differences between lattice and continuum in the small $R$ regime, the general prospects of the investigation of compactified QCD (adj) on the lattice are quite good. Even the phase with strong deviations between lattice and continuum the theory remains confined. The region where the properties of the theory are most similar to the continuum expectation is close to the peak of the susceptibility. At this point the nearly flat effective potential provides an ideal playground to investigate non-perturbative semiclassical effects.

Already the case of the $N_{f}=2 \mathrm{QCD}$ (adj) is quite difficult to investigate due to the near conformal behaviour. Our observations show a deconfined intermediate phase, but a more precise analysis is required to investigate the large volume and small mass regime of this theory. The interest to this theory is also related to the determination of possible candidates for a walking technicolour scenario.

In addition our investigations are related to the considerations of supersymmetric YangMills theory compactified to lower space times. In some of these investigations the compactification is done on the lattice setting $N_{t}=1$ in the compacitified direction. From the perspective of our investigations, this might lead to sizable deviations from the theory that is compactified in the continuum. For example a three dimensional supersymmetric Yang-Mills theory obtained from simulations at $R / a=1$ would be in the confined lattice perturbative phase that we have observed.

Finally, in the near future we plan to investigate whether the same confining effects occur when the theory is compactified down to two dimensions.

\section{Acknowledgments}

The authors gratefully acknowledge the Gauss Centre for Supercomputing e.V. (www.gauss-centre.eu) for funding this project by providing computing time on the GCS Supercomputer SuperMUC at Leibniz Supercomputing Centre (LRZ, www.lrz.de). We thank Tin Sulejmanpasic for collaboration in early stages of this work, Misha Shifman and Aleksey Cherman for many discussions on Abelian vs. non-Abelian confinement. We thank 
Gernot Münster, Istvan Montvay, and Philipp Scior for helpful comments on the draft. G.B. acknowledges support from the Deutsche Forschungsgemeinschaft (DFG) Grant No. BE 5942/2-1. M. Ü. acknowledges support from U.S. Department of Energy, Office of Science, Office of Nuclear Physics under Award Number DE-FG02-03ER41260.

Open Access. This article is distributed under the terms of the Creative Commons Attribution License (CC-BY 4.0), which permits any use, distribution and reproduction in any medium, provided the original author(s) and source are credited.

\section{References}

[1] A.M. Polyakov, Quark Confinement and Topology of Gauge Groups, Nucl. Phys. B 120 (1977) 429 [INSPIRE].

[2] I. Affleck, J.A. Harvey and E. Witten, Instantons and (Super)Symmetry Breaking in (2+1)-Dimensions, Nucl. Phys. B 206 (1982) 413 [InSPIRE].

[3] M. Ünsal, Abelian duality, confinement and chiral symmetry breaking in QCD(adj), Phys. Rev. Lett. 100 (2008) 032005 [arXiv:0708.1772] [INSPIRE].

[4] M. Ünsal, Magnetic bion condensation: A New mechanism of confinement and mass gap in four dimensions, Phys. Rev. D 80 (2009) 065001 [arXiv:0709.3269] [INSPIRE].

[5] M. Ünsal and L.G. Yaffe, Center-stabilized Yang-Mills theory: Confinement and large N volume independence, Phys. Rev. D 78 (2008) 065035 [arXiv:0803.0344] [INSPIRE].

[6] M. Shifman and M. Ünsal, QCD-like Theories on $R_{3} \times S_{1}$ : A Smooth Journey from Small to Large $r\left(S_{1}\right)$ with Double-Trace Deformations, Phys. Rev. D 78 (2008) 065004 [arXiv:0802.1232] [INSPIRE].

[7] N. Seiberg and E. Witten, Gauge dynamics and compactification to three-dimensions, in The mathematical beauty of physics: A memorial volume for Claude Itzykson, proceedings, conference, Saclay, France, 5-7 June 1996, pp. 333-366, (1996), hep-th/9607163 [INSPIRE].

[8] K.-M. Lee and P. Yi, Monopoles and instantons on partially compactified D-branes, Phys. Rev. D 56 (1997) 3711 [hep-th/9702107] [INSPIRE].

[9] T.C. Kraan and P. van Baal, Periodic instantons with nontrivial holonomy, Nucl. Phys. B 533 (1998) 627 [hep-th/9805168] [INSPIRE].

[10] N.M. Davies, T.J. Hollowood, V.V. Khoze and M.P. Mattis, Gluino condensate and magnetic monopoles in supersymmetric gluodynamics, Nucl. Phys. B 559 (1999) 123 [hep-th/9905015] [INSPIRE].

[11] P.C. Argyres and M. Ünsal, The semi-classical expansion and resurgence in gauge theories: new perturbative, instanton, bion and renormalon effects, JHEP 08 (2012) 063 [arXiv: 1206.1890] [INSPIRE].

[12] E. Poppitz and T. Sulejmanpasic, (S)QCD on $\mathbb{R}^{3} \times \mathbb{S}^{1}$ : Screening of Polyakov loop by fundamental quarks and the demise of semi-classics, JHEP 09 (2013) 128 [arXiv: 1307.1317] [INSPIRE].

[13] G.V. Dunne and M. Ünsal, Generating nonperturbative physics from perturbation theory, Phys. Rev. D 89 (2014) 041701 [arXiv:1306.4405] [InSPIRE]. 
[14] G.V. Dunne and M. Ünsal, New Nonperturbative Methods in Quantum Field Theory: From Large-N Orbifold Equivalence to Bions and Resurgence, Ann. Rev. Nucl. Part. Sci. 66 (2016) 245 [arXiv: 1601.03414] [INSPIRE].

[15] G.V. Dunne and M. Ünsal, Resurgence and Trans-series in Quantum Field Theory: The CP(N-1) Model, JHEP 11 (2012) 170 [arXiv:1210.2423] [INSPIRE].

[16] A. Cherman, D. Dorigoni and M. Ünsal, Decoding perturbation theory using resurgence: Stokes phenomena, new saddle points and Lefschetz thimbles, JHEP 10 (2015) 056 [arXiv: 1403.1277] [INSPIRE].

[17] D.J. Gross, R.D. Pisarski and L.G. Yaffe, $Q C D$ and Instantons at Finite Temperature, Rev. Mod. Phys. 53 (1981) 43 [inSPIRE].

[18] E. Witten, Constraints on Supersymmetry Breaking, Nucl. Phys. B 202 (1982) 253 [INSPIRE].

[19] E. Poppitz, T. Schäfer and M. Ünsal, Continuity, Deconfinement and (Super) Yang-Mills Theory, JHEP 10 (2012) 115 [arXiv:1205.0290] [INSPIRE].

[20] Y. Hosotani, Dynamical Mass Generation by Compact Extra Dimensions, Phys. Lett. B 126 (1983) 309 [INSPIRE].

[21] P. Kovtun, M. Ünsal and L.G. Yaffe, Volume independence in large $N_{c}$ QCD-like gauge theories, JHEP 06 (2007) 019 [hep-th/0702021] [INSPIRE].

[22] S. Coleman, Aspects of Symmetry: Selected Erice Lectures, Cambridge University Press, Cambridge (1985).

[23] T. Schäfer and E.V. Shuryak, Instantons in QCD, Rev. Mod. Phys. 70 (1998) 323 [hep-ph/9610451] [INSPIRE].

[24] M.M. Anber and E. Poppitz, Two-flavor adjoint QCD, Phys. Rev. D 98 (2018) 034026 [arXiv:1805.12290] [INSPIRE].

[25] M.M. Anber, E. Poppitz and B. Teeple, Deconfinement and continuity between thermal and (super) Yang-Mills theory for all gauge groups, JHEP 09 (2014) 040 [arXiv:1406.1199] [INSPIRE].

[26] H. Kouno, T. Misumi, K. Kashiwa, T. Makiyama, T. Sasaki and M. Yahiro, Differences and similarities between fundamental and adjoint matters in $\mathrm{SU}(N)$ gauge theories, Phys. Rev. D 88 (2013) 016002 [arXiv:1304.3274] [INSPIRE].

[27] K. Kashiwa and T. Misumi, Phase structure and Hosotani mechanism in gauge theories with compact dimensions revisited, JHEP 05 (2013) 042 [arXiv: 1302.2196] [INSPIRE].

[28] M.M. Anber, S. Collier, E. Poppitz, S. Strimas-Mackey and B. Teeple, Deconfinement in $\mathcal{N}=1$ super Yang-Mills theory on $\mathbb{R}^{3} \times \mathbb{S}^{1}$ via dual-Coulomb gas and "affine" $X Y$-model, JHEP 11 (2013) 142 [arXiv:1310.3522] [INSPIRE].

[29] G. Bergner and S. Piemonte, Compactified $\mathcal{N}=1$ supersymmetric Yang-Mills theory on the lattice: continuity and the disappearance of the deconfinement transition, JHEP 12 (2014) 133 [arXiv: 1410.3668] [INSPIRE].

[30] I. Montvay and G. Münster, Quantum fields on a lattice, Cambridge University Press (1994).

[31] G. Bergner, P. Giudice, G. Münster, I. Montvay and S. Piemonte, The light bound states of supersymmetric SU(2) Yang-Mills theory, JHEP 03 (2016) 080 [arXiv:1512.07014] [INSPIRE]. 
[32] G. Bergner, I. Montvay, G. Münster, U.D. Özugurel and D. Sandbrink, Towards the spectrum of low-lying particles in supersymmetric Yang-Mills theory, JHEP 11 (2013) 061 [arXiv: 1304.2168] [INSPIRE].

[33] G. Münster and H. Stüwe, The mass of the adjoint pion in $\mathcal{N}=1$ supersymmetric Yang-Mills theory, JHEP 05 (2014) 034 [arXiv:1402.6616] [INSPIRE].

[34] G. Bergner, P. Giudice, G. Münster, S. Piemonte and D. Sandbrink, Phase structure of the $\mathcal{N}=1$ supersymmetric Yang-Mills theory at finite temperature, JHEP 11 (2014) 049 [arXiv: 1405.3180] [INSPIRE].

[35] A.M. Ferrenberg, J. Xu and D.P. Landau, Pushing the limits of Monte Carlo simulations for the three-dimensional Ising model, Phys. Rev. E 97 (2018) 043301 [arXiv:1806.03558] [INSPIRE].

[36] B. Bringoltz, Large- $N$ volume reduction of lattice $Q C D$ with adjoint Wilson fermions at weak-coupling, JHEP 06 (2009) 091 [arXiv: 0905.2406] [INSPIRE].

[37] E. Poppitz and M. Ünsal, Comments on large- $N$ volume independence, JHEP 01 (2010) 098 [arXiv:0911.0358] [INSPIRE].

[38] M. Ünsal and L.G. Yaffe, Large-N volume independence in conformal and confining gauge theories, JHEP 08 (2010) 030 [arXiv: 1006.2101] [INSPIRE].

[39] G. Cossu, H. Hatanaka, Y. Hosotani and J.-I. Noaki, Polyakov loops and the Hosotani mechanism on the lattice, Phys. Rev. D 89 (2014) 094509 [arXiv: 1309.4198] [INSPIRE].

[40] H. Georgi and S.L. Glashow, Unified weak and electromagnetic interactions without neutral currents, Phys. Rev. Lett. 28 (1972) 1494 [INSPIRE].

[41] M.M. Anber, The abelian confinement mechanism revisited: new aspects of the Georgi-Glashow model, Annals Phys. 341 (2014) 21 [arXiv: 1308.0027] [InSPIRE].

[42] N. Seiberg and E. Witten, Electric-magnetic duality, monopole condensation and confinement in $N=2$ supersymmetric Yang-Mills theory, Nucl. Phys. B 426 (1994) 19 [Erratum ibid. B 430 (1994) 485] [hep-th/9407087] [INSPIRE].

[43] G. 't Hooft, Topology of the Gauge Condition and New Confinement Phases in Nonabelian Gauge Theories, Nucl. Phys. B 190 (1981) 455 [inSPIRE].

[44] M.R. Douglas and S.H. Shenker, Dynamics of SU(N) supersymmetric gauge theory, Nucl. Phys. B 447 (1995) 271 [hep-th/9503163] [INSPIRE].

[45] E. Poppitz and M.E. Shalchian T., String tensions in deformed Yang-Mills theory, JHEP 01 (2018) 029 [arXiv: 1708.08821] [inSPIRE].

[46] L. O'Raifeartaigh, A. Wipf and H. Yoneyama, The Constraint Effective Potential, Nucl. Phys. B 271 (1986) 653 [INSPIRE].

[47] C.P. Korthals Altes, Constrained effective potential in hot QCD, Nucl. Phys. B 420 (1994) 637 [hep-th/9310195] [INSPIRE].

[48] A. Dumitru, Y. Guo and C.P. Korthals Altes, Two-loop perturbative corrections to the thermal effective potential in gluodynamics, Phys. Rev. D 89 (2014) 016009 [arXiv: 1305.6846] [INSPIRE].

[49] A. Athenodorou, E. Bennett, G. Bergner and B. Lucini, Infrared regime of SU(2) with one adjoint Dirac flavor, Phys. Rev. D 91 (2015) 114508 [arXiv:1412.5994] [InSPIRE]. 
[50] G. Bergner and S. Piemonte, Running coupling from gluon and ghost propagators in the Landau gauge: Yang-Mills theories with adjoint fermions, Phys. Rev. D 97 (2018) 074510 [arXiv: 1709.07367] [INSPIRE].

[51] D. Smith, A. Dumitru, R. Pisarski and L. von Smekal, Effective potential for SU(2) Polyakov loops and Wilson loop eigenvalues, Phys. Rev. D 88 (2013) 054020 [arXiv:1307.6339] [INSPIRE].

[52] C. Gattringer, Linking confinement to spectral properties of the Dirac operator, Phys. Rev. Lett. 97 (2006) 032003 [hep-lat/0605018] [INSPIRE].

[53] G. Cossu and M. D'Elia, Finite size phase transitions in QCD with adjoint fermions, JHEP 07 (2009) 048 [arXiv:0904.1353] [INSPIRE]. 